\title{
1 Chimeric RNA:DNA Donorguide Improves HDR in vitro and in vivo
}

2 Brandon W. Simone ${ }^{1}$, Han B. Lee ${ }^{1}$, Camden L. Daby ${ }^{1}$, Santiago Restrepo-Castillo², Gabriel Martínez-

3 Gálvez ${ }^{3}$, Hirotaka Ata ${ }^{4}$, William A.C. Gendron², Karl J. Clark ${ }^{1}$, Stephen C. Ekker ${ }^{1^{*}}$

$4 \quad{ }^{1}$ Department of Biochemistry and Molecular Biology, Mayo Clinic, Rochester, MN

$5 \quad{ }^{2}$ Mayo Clinic Graduate School of Biomedical Sciences, Virology and Gene Therapy Track, Mayo Clinic,

6 Rochester, MN

$7 \quad{ }^{3}$ Mayo Clinic Graduate School of Biomedical Sciences, Biomedical Engineering and Physiology Track,

8 Mayo Clinic, Rochester, MN

$9 \quad{ }^{4}$ Department of Clinical and Translational Sciences, Mayo Clinic, Rochester, MN

10

*Corresponding author: Stephen C. Ekker- Ekker.Stephen@mayo.edu, Department of Biochemistry and

11 Molecular Biology, Mayo Clinic, 200 1st 10 Street SW, Rochester, MN 55905.

12 ORC ID:

13 BWS-0000-0003-3907-3015; HBL-0000-0002-6633-966X; CLD-0000-0002-2121-4927 ; SRC-- 0000-0001-

14 7881-1813; GMG- 0000-0002-4392-2596; HA-0000-0003-0027-205X; WAG- 0000-0002-2280-3133; KJC-

15

0000-0002-9637-0967; SCE- 0000-0003-0726-4212

16 


\section{Abstract}

Introducing small genetic changes to study specific mutations or reverting clinical mutations to wild type has been an area of interest in precision genomics for several years. In fact, it has been found that nearly $90 \%$ of all human pathogenic mutations are caused by small genetic variations, and the methods to efficiently and precisely correct these errors are critically important. One common way to make these small DNA changes is to provide a single stranded DNA (ssDNA) donor containing the desired alteration together with a targeted double-strand break (DSB) at the genomic target. The donor is typically flanked by regions of homology that are often $\sim 30-100 \mathrm{bp}$ in length to leverage the homology directed repair (HDR) pathway. Coupling a ssDNA donor with a CRISPR-Cas9 to produce a targeted DSB is one of the most streamlined approaches to introduce small changes. However, in many cell types this approach results in a low rate of incorporation of the desired alteration and has undesired imprecise repair at the $5^{\prime}$ or $3^{\prime}$ junction due to artifacts of the DNA repair process. We herein report a technology that couples the spatial temporal localization of an ssDNA repair template and leverages the nucleic acid components of the CRISPR-Cas9 system. We show that by direct fusion of an ssDNA template to the trans activating RNA (tracrRNA) to generate an RNA-DNA chimera, termed Donorguide, we recover precise integration of genetic alterations, with both increased integration rates and decreased imprecision at the 5' or 3' junctions relative to an SSODN donor in vitro in HEK293T cells as well as in vivo in zebrafish. Further, we show that this technology can be used to enhance gene conversion with other gene editing tools such as TALENs. 


\section{Introduction}

Targeted genomic alterations have revolutionized the field of molecular biology. Most recently, these efforts have been focused on designer nucleases such as the clustered regular interspersed short palindromic repeat (CRISPR) and their corresponding CRISPR associated proteins (Cas), namely Cas9 ${ }^{12} 3$.

These Cas proteins can be directed to a specific region in the genome specified by an 20nt guide RNA and induce a targeted double-strand break $^{3}$. In the absence of a repair template, the endogenous DNA repair machinery will most likely repair the lesion by re-ligating the free DNA ends by an un-templated repair pathway such as non-homologous end joining (NHEJ) ${ }^{4}$ or alternative end joining (Alt-EJ) including

47 microhomology-mediated end joining (MMEJ) ${ }^{56}$. In the presence of a repair template such as a single stranded oligo deoxy nucleotide (ssODN) or a double stranded DNA (dsDNA) template in the form of a PCR product or plasmid, precise repair is triggered that is directed by regions of homology on the donor template that mimic endogenous DNA repair using a chromosome as a repair template ${ }^{7}$. Gene replacement via dsDNA template has been shown to be RAD-51 dependent ${ }^{89}$ and be active in G2/M of the cell cycle when a homologous chromosomal template would be present ${ }^{10}$. Conversely, single stranded- template repair (SSTR) has been shown to be RAD-51 independent ${ }^{11}$ and be inactive during $\mathrm{G} 2 / \mathrm{M}^{12}$. Further, SSTR has recently been found to be dependent on the Fanconi Anemia (FA) pathway ${ }^{7}$.

Repair with a dsDNA donor is relatively inefficient in most cell types ${ }^{13}$. Alternatively, repair via an ssDNA donor has been shown to be efficient for small genetic changes in multiple cell types ${ }^{14}{ }^{13}{ }^{15}$. Several

57 studies have demonstrated that ssODNs are sufficient donors for inserting small $(<50 \mathrm{bp})$ changes into

58 the genome with appreciable rates ${ }^{16}$. Although many gene replacement strategies with dsDNA use long 59 homology arms of $0.5 \mathrm{kbp}-1 \mathrm{kbp}$, ssDNA-mediated gene replacement can proceed with homologies much 60 shorter than this ${ }^{17}$. In fact, the length of flanking homologies has been shown to be decreasingly

61 important with the insertion of smaller cargos into the genome ${ }^{18}{ }^{17}$. For insertion of smaller cargos such 
62 as single base pair insertions or substitutions, single stranded oligos with at least 30bp of flanking

63 homology are typically employed.

64 Though it is a relatively efficient process in vitro, rates of homology directed repair (HDR) with Cas9-

65 mediated double strand breaks (DSBs) coupled with an SsODN repair template in zebrafish are typically

66 quite modest ${ }^{19} 20$ and often result in imprecise repair at either the $5^{\prime}$ or $3^{\prime}$ junction that is thought to be

67 an artifact of the DNA repair process ${ }^{21}$. In zebrafish, the imprecision at the insertion junctions has been

68 explained, at least partly, by the stochastic integration of fragments of the ssODN repair template

$69 \quad 22$. Though ssODN template induced changes are typically sufficient for studying a clinically relevant

70 mutation or generating a transgenic cell line, the low efficiency and imprecise nature of the insertion

71 events can render this methodology difficult to deploy in applications that require single base-pair

72 precision.

73 Recent work has shown that the spatial-temporal localization of a repair template via covalent linkage of

74 an ssDNA donor to the Cas9 nuclease increases rates of HDR significantly ${ }^{23}{ }^{24}$. However, these

75 approaches require complex protein-nucleic acid engineering for each target locus and therefore

76 preclude them from being widely adapted approaches for precision gene engineering. These

77 observations using a protein-nucleic acid fusion indicate that methods that improve spatial temporal

78 location of the repair template increase rates of gene replacement.

79 Several methods have been developed to preclude the need for the traditional targeted DSB coupled

80 with a homologous repair template, including base editing (BE) ${ }^{25,26}$ and prime editing (PE) ${ }^{27}$. Though

81 these technologies do not require a DSB followed by HDR with a DNA repair template, they are not

82 without their drawbacks. Even with several iterations of the BE system, the editing window remains

83 relatively large and makes high throughput and precision applications difficult. Additionally, prime 
84 editing requires several large and potentially toxic components to be delivered to the system and relies

85 on successful delivery and activity of a viral reverse transcriptase.

86 Here we report a methodology that is backwards-compatible with common CRISPR technologies

87 available to most research laboratories that uses the spatial-temporal components of the CRISPR-Cas9

88 system and small flanking homologies sufficient for small DNA modifications. We demonstrate that by

89 direct covalent fusion of an SSDNA repair template to the tracrRNA, deliverable as CRISPR-Cas9 nuclease

90 RNP results in precise gene conversion with as little as 10bp of homology when coupled with a CRISPR-

91 Cas9 mediated DSB. We demonstrate that our approach is amenable both in vitro as well as in vivo using

92 human embryonic kidney (HEK293T) cells and zebrafish, respectively. Additionally, we find that

93 Donorguide can enhance the activity of other gene editing tools such as TALENs to increase gene

94 modification. Taken together this work provides a streamlined approach that requires only a single RNP

95 complex be delivered to induce precise molecular alterations at rates comparable to the imprecise

96 repair of ssODN-mediated HDR.

97 


\section{Results}

101 To assess the feasibility of covalently tethering a DNA template to the $3^{\prime}$ end of a tracrRNA to induce

102 precise genetic edits, we employed the HEK 293T cell system due to their ease of transfection (Figure 1).

103 We previously identified a highly active Streptococcus pyogenes Cas9 (SpCas9) target site in the safe

104 harbor region AAVS1 ${ }^{28}$ (Supplemental Figure 1 ) and determined this to be a candidate locus for our 105 approach to ensure that SpCas9 was active at the target locus. Due to synthetic RNA synthesis limits, we 106 used the separate CRISPR RNA (crRNA) tracrRNA system instead of the single guide RNA (sgRNA) system.

107 We fused a DNA repair template to the $3^{\prime}$ end of the tracrRNA containing $12 \mathrm{bp}$ of homology flanking a

1082 2bp insertion (Fig 2a) generating a chimeric RNA:DNA hybrid. This chimeric tracrRNA was annealed with

109 a canonical SpCas9 crRNA to form what we term the Donorguide and combined with SpCas9

110 recombinant protein to form the active RNP complex. HEK 293T cells were electroporated with the

111 SpCas9 Donorguide RNP and screened for genetic insertions 48 hours later by next generation

112 sequencing (Fig 1). We found that the Donorguide SpCas9 RNP introduced the precise 2bp insertion at

$113 \sim 2 \%$ efficiency (Fig 2B). In addition, we found that Donorguide introduced indel mutations at markedly

114 lower rates than a canonical tracrRNA. These data suggested that the Donorguide methodology is a

115 viable approach to introduce precise small molecular changes in vitro, albeit at modest rates.

Donorguide SpCas9 introduces insertions more efficiently than a separate ssODN donor in vivo

117 Encouraged by our in vitro results we sought to see if our results could be generalized to in vivo animal

118 models. We chose the zebrafish model due to their high fecundity, amenability to microinjection, and

119 ease of genotyping (Figure 1). Single cell zebrafish embryos were either injected with preformed SpCas9

120 RNP with a crRNA targeting the $N 2 B$ gene in the Titin locus that we have previously shown to be highly

121 active ${ }^{6}$ (Fig 3a) along with separate "free floating" ssODN donor providing a 2 nt insertion or as a CRISPR 
122 Donorguide containing the same 2 nt insertion. Injected animals were incubated for 72 hours before

123 having their genomic DNA isolated. Injected animal genomic DNA was pooled, PCR amplified and

124 submitted for Next Generation Sequencing (Fig 1). We found that using Donorguide SpCas9, compared

125 to WT Cas9 and a free ssODN donor, results in a similar rate of overall HDR events (Average $9.27 \% \pm 4.34$

126 for ssODN and $12.64 \% \pm 2.97$ for Donorguide) comprised of both precise and imprecise insertion events

127 (Fig 3B). However, the genetic alterations induced by Donorguide SpCas9 $(5.85 \% \pm 1.18)$ resulted in a

128 higher rate of precise insertions when compared to a free floating ssODN donor (3.44\% \pm 2.72$)$ (Fig 3B)

Donorguide SpCas9 introduces small molecular changes more efficiently than a separate ssODN donor in

$\underline{\text { vitro }}$

Having shown that Donorguide is suitable to introduce small insertions in vitro we set out to assess if

132 Donorguide could likewise introduce precise genetic substitutions. To this end we employed a previously established EGFP-BFP reporter system ${ }^{29}$ to fluorometrically measure genetic substitution. EGFP contains converts it to BFP, which can be recorded by flow cytometry (Fig 4a). HEK293T cells were transfected with an EGFP expressing plasmid that is flanked by Tol2 inverted terminal repeats (ITRs) as well as a

137 plasmid encoding tol2 transposase. Cells were passaged several times and were sorted for the brightest 138 EGFP expression cells to establish the stable reporter cell line, termed HEK-GFP (Supplemental Figure 2).

HEK-GFP cells were transfected with Donorguide or a standard guide with a "free floating" ssODN donor convert EGFP to BFP, and this region was flanked by 10nt of homology on either side (Fig 4B). Cells were incubated at $37^{\circ} \mathrm{C}$ for 72 hours following electroporation and BFP expression was measured by 
Donorguide converted EGFP to BFP at a significantly greater rate (average $\sim 1.7 \% \pm 0.48 \mathrm{BFP}+$ ) as

146 measured by flow cytometry (Fig 4 C-D) and next generation sequencing (Supplemental Figure 3). These

147 results suggest that when compared to a free SSODN of similar size, CRISPR Donorguide results in a 148 quantitative improvement of HDR induction in vitro.

Donorguide SpCas9 introduces small molecular substitutions more efficiently than a separate ssODN

To assess if the conversion of EGFP-BFP observed in our HEK-GFP reporter system translated in vivo, we utilized a previously established transgenic zebrafish line that has an EGFP reporter stably integrated

154 into its genome ${ }^{30}$ (Fig 5A). Single cell zebrafish embryos were injected with either WT SpCas9 with an

155 ssODN encoding the EGFP-BFP transition or CRISPR Donorguide carrying the transition. The injected

156 animals were pooled, had their DNA isolated, barcoded, and submitted for Next Generation Sequencing.

157 We found that compared to a free sSODN, CRISPR Donorguide $(1.68 \% \pm 1.25)$ results in more precise

158 insertion events compared to an ssODN of a similar size $(0.13 \% \pm 0.17)$ (Fig $5 \mathrm{~B})$. These results suggest that

159 CRISPR Donorguide functions cross-model to enhance HDR relative to an ssODN.

Donorguide SpCas9 introduces insertions more efficiently than a separate ssODN donor in vitro

161 Encouraged by our in vivo results, we sought to determine if the size of the homology arms explained

162 the modest rates of HDR with CRISPR Donorguide in vitro (Fig 2B). To address this, we increased the 163 homology arm length to 23nt for both CRISPR Donorguide and its requisite sSODN designed to introduce

164 a 2nt insertion at AAVS1 (Fig 5A). The larger 23-2-23 Donorguide or WT SpCas9 and ssODN were 165 electroporated into HEK293T cells, incubated for 72 hours, and analyzed for HDR by Next Generation 
precise HDR rates approaching 30\% with CRISPR Donorguide and these rates were significantly greater than Cas9 and a standard ssODN donor that resulted in approximately $10 \%$ precise HDR (Fig 5b). These data suggest that CRISPR Donorguide can introduce HDR at appreciable rates and outperform a standard ssODN DNA donor.

\section{Donorguide SpCas9 introduces a greater proportion of desired mutant alleles than a separate ssODN}

172 donor in vitro

173 Small insertions comprise about 3\% of known human pathogenic genetic variants ${ }^{27}$ and being able to 174 correct these aberrant sequences to restore the wild type gene is highly sought after. Targeting these insertion regions with CRISPR/Cas9 to resolve the lesion can result in additional loss of function alleles as a result of NHEJ or Alt-EJ repair. Therefore, precise deletions are required to address such conditions. rates than a comparable ssODN. To assess precise deletion, we developed an EGFP-STOP-mRFP reporter cell line such that unperturbed cells will be EGFP+, but if a precise deletion of the stop codon is introduced the cells will be EGFP+/mRFP+ (Fig 7a). EGFP-STOP-mRFP cells were transfected with either SpCas9 and an ssODN encoding the 3nt stop codon deletion or with CRISPR Donorguide likewise

183 containing the deletion, incubated for 72 hours, and assessed the induction of the deletion by NGS. We 184 found that CRISPR Donorguide introduced the precise deletion at nearly the same rate as a traditional ssODN (11.45\% \pm 3.45 ssODN and 10.55\% \pm 4.03 Donorguide) . However, we found that, in agreement with previous observations (Figure 2B) that Donorguide resulted in a significantly decreased amount of

187 overall indel mutations compared to SpCas9 ( $88.77 \% \pm 9.80$ ssODN $41.23 \% \pm 14.61$ Donorguide).

188 Accordingly, CRISPR Donorguide resulted in a significantly greater percentage of precise deletion alleles, 
$12.73 \pm 2.95$ ssODN). These results suggest that CRISPR Donorguide can introduce a precise deletion at a comparable rate to an ssODN, but the overall fraction of desired alleles is significantly greater in the mutant allele population.

Donorguide SpCas9 introduces a greater proportion of desired mutant alleles than a separate ssODN donor in vivo

As Donorguide generated a greater proportion of mutant alleles in vitro, we tested whether a similar phenomenon was also applicable in vivo. We developed an assay to effectively remove WT alleles from the population while simultaneously interrogating whether the remaining mutant alleles were generated by stochastic NHEJ-mediated indels or by a precise molecular alteration. To this end we targeted the tyrosinase (tyr) gene in zebrafish, which is required for converting tyrosine into the pigment melanin that confers skin color ${ }^{31}$. We inserted a 6 nt Avrll restriction site with either an ssODN or CRISPR Donorguide into the tyr locus (Figure 9A). If precisely inserted, the cargo will generate an inframe stop codon and should lead to the development of albino zebrafish mutants (Figure 9B). The resulting albino zebrafish from both conditions were then pooled, had their genomic DNA isolated, and were PCR amplified. The resulting PCR amplicons were sub-cloned into a TA cloning vector and successfully generated TA clones were identified by blue-white screening. The resulting subclones from both the ssODN and Donorguide conditions were subjected to colony PCR followed by restriction fragment length polymorphism (RFLP) analysis using Avrll followed by agarose gel electrophoresis to visualize successfully digested (and therefore successfully integrated) alleles (Figure 9G).

Interestingly, while Cas9 and the ssODN donor generated albino mutants (Figure 9D) we observed only wild type looking zebrafish in the Donorguide condition (Figure 9E). Upon further inspection, we found 
that the DNA portion of the tyr Donorguide was designed complementary to the crRNA target strand.

214 We reasoned that the DNA region of the Donorguide and the crRNA were aberrantly annealing during

215 Donorguide assembly and preventing the formation of the crRNA:Donorguide duplex and therefore the

216 subsequent RNP complex. We then designed the tyr Donorguide DNA region to be of the same strand as

217 the crRNA target strand, and therefore prevent aberrant crRNA:Donorguide base pairing. Upon

218 switching the orientation of the DNA donor portion, we observed strong albino phenotypes using the

219 same strand Donorguide (Figure 9F). After performing the RFLP analysis we found that just $25 \%$ of the

220 ssODN clones (10/40) were successfully digested with Avrll. However, we found that over $80 \%$ of the

221 Donorguide clones (34/41) were successfully digested with Avrll. These results suggest that when

222 eliminating WT alleles by phenotypic screening, CRISPR Donorguide comprises a majority of the mutant

223 alleles, whereas SpCas9 and an ssODN generate mostly stochastic indel mutations.

226 Even with the advent of CRISPR orthologous such as Cas12a ${ }^{32}$ and enhanced CRISPR Cas9 variants with

227 decreased PAM stringency ${ }^{33}$, there is still a PAM and gRNA requirement that could preclude the

228 utilization of CRISPR tools. This is particularly an issue when trying to perform HDR, where it has been

229 demonstrated that gene conversion is greatly enhanced when the genetic change is introduced more

230 proximally to the site of the DNA DSB ${ }^{34}$. TALENs (transcription activation like effector nucleases) on the

231 other hand, have only a 5' thymidine requirement, which can also be removed with mutagenic variants

$232 \quad{ }^{35}{ }^{36}$. TALENs have been shown to function efficiently as gene editing tools in vivo ${ }^{37} 38$ and in vitro ${ }^{39}$ for

233 both gene knockout and knock-in ${ }^{40}$.

234 To this end, we wanted to assess if Donorguide could enhance TALEN-mediated HDR. We designed

235 TALENs targeting EGFP using our previously established FusX assembly protocol (Supplemental Table 1) 
$236{ }^{41}$. We then designed a catalytically inactive Cas9 (dCas9) Donorguide targeting the EGFP chromophore

237 domain $80 \mathrm{bp}$ downstream of the TALEN target site to prevent steric interference between dCas9 and

238 the right TALEN arm. HEK-GFP cells were transfected with both the EGFP-TALENs and an sSODN or with

239 dCas9 Donorguide containing the EGFP-BFP transition (Fig 7a) and measured for BFP+ cells 72 hours post

240 transfection by flow cytometry. Strikingly, we found that TALENs with dCas9 Donorguide resulted in an

241 approximate 3-fold increase in BFP+ cells compared to TALENs with an ssODN donor. Taken together,

242 these data suggest that CRISPR Donorguide results in greater gene conversion than a traditional ssODN

243 donor and can be used in concert with non-Cas9 gene editing tools such as TALENs. 


\section{Discussion}

In this work we report a facile approach to introduce small genetic changes in vitro in HEK293T cells as

well as in vivo in zebrafish. These results suggest that this methodology can be rapidly adapted to other cell types of interest to study protein function, validate variants of uncertain significance (VUSs), or generate isogenic cell populations to assess the implications of a patient mutation. We further show that this approach is amenable to in vivo editing in the zebrafish model. The success of this approach in zebrafish further suggests that this technology can be readily translated to other in vivo models such as mice, rats, and larger animal models. using our chimeric guide approach. In zebrafish, our data suggest that for small genetic modifications it

258 would be preferable to use the Donorguide approach to increase both the overall amount and precision 259 of gene integration events. This result enables researchers to better make animal models and should require less screening for allelic alterations. Imprecision of insertion events in vivo is well documented in

261 the field ${ }^{42} 434$ and has been partially explained by several phenomenon, but a unifying explanation has

262 been largely elusive. We observe genetic imprecision in zebrafish in agreement with previous reports of

263 using ssODN donors ${ }^{44}$. We find that using Donorguide at least partially ameliorates some of the

264 imprecision observed with ssODN donors. This could be explained, at least in part, by one of two factors.

265 First, the nature of the DNA-RNA hybrid of the Donorguide could be less accessible to cellular nucleases

266 and reduce the presence of donor fragments aberrantly integrating into the genomic lesion. Second, the

267 Donorguide could be physically protected by the Cas9 RNP complex likewise sparing it from cellular

268 exonucleases that ssODNs are otherwise susceptible to similarly reducing the presence of donor DNA 269 fragments. 
Conversely, there are limited reports of such imprecision in mammalian systems. Accordingly, we

271 observe little imprecision in our in vitro assays. We find that in our in vitro studies the improvement of

272 HDR with Donorguide compared to an SSODN is primarily an overall increase in desired mutant alleles

273 without a significant change in precision of the events. We note one exception to this net increase of

274 HDR events with Donorguide in our small deletion study. While we find that the net amount of precise

275 insertion events is comparable between the ssODN and Donorguide, our approach results in a greater

276 proportion of desired alterations in the pool of mutant alleles in vitro.

277 We recapitulated this increased mutant allele proportion phenomena at the tyr locus in zebrafish and

278 showed significantly more insertion events in the Donorguide condition compared to the ssODN

279 condition. It is worth mentioning that though there were significantly more Avrll digested clones using

280 Donorguide ( $82 \%$ vs. $25 \%$ with ssODN), many of the PCR products were not completely digested

281 (Supplemental Figure 4), suggesting that they may not be precise insertion events. Indeed, when we

performed Sanger sequencing on several ssODN and Donorguide digested subclones we found a mix of both precise and imprecise insertion events.

Being able to narrow down the editing outcomes to either a desired alteration or no editing at all may

286 formation of sickled hemoglobin ${ }^{45}$. Though the sickled hemoglobin is causal for the pathology of SCD,

287 total loss of function introduced to correct the point mutation would likely be more harmful than the

288 original point mutant. In SCD and similar pathologies, Donorguide would therefore be a preferred

289 method of correction in which the genetic outcome is more likely to be no editing at all or a precise

290 alteration with less collateral genetic damage.

291 In our in vitro studies we found that increasing the lengths of homology flanking the insertion resulted in 292 a marked increase in HDR events. At this time, we are generating these chimeric tracrRNA molecules 
from commercial manufacturers. While it is of interest to systematically increase the length of the DNA for 50 nt of DNA be added to the $3^{\prime}$ end of the tracrRNA, currently limiting the possibility of cargos and lengths of flanking homology. In-house synthesis of these chimeric tracrRNA molecules could afford the

297 addition of larger homology arms flanking diverse cargos such as peptide epitope tags or fluorescent reporter genes. zebrafish, 12 nt of homology flanking a cargo of 2-6nt should be a starting point for Donorguide design. be the starting point. 
bioRxiv preprint doi: https://doi.org/10.1101/2021.05.28.446234; this version posted May 28, 2021. The copyright holder for this preprint (which

was not certified by peer review) is the author/funder, who has granted bioRxiv a license to display the preprint in perpetuity. It is made available under aCC-BY-NC 4.0 International license.

316 applications could therefore leverage Donorguide for its desired editing outcomes. We likewise include

317 design guidelines to deploy CRISPR Donorguide in their respective model systems for enhanced

318 precision genome editing applications. 


\section{Methods}

\section{Cell culture}

323 HEK293T cells were obtained from ATCC (CRL-3216). Cells were maintained in Dulbecco's modified

324 Eagle's medium (Gibco \#11995-040) supplemented with 10\% fetal bovine serum (Gibco \#26140079) and 325 1\% penicillin/streptomycin (Gibco \#15140-122).

\section{In vitro Donorguide RNP preparation}

328 Protocol adapted from iPS cell electroporation-NepaGene. Briefly, Alt-R CRISPR-Cas9 crRNAs were ordered from IDT ( $\underline{w w w . i d t d n a . c o m / C R I S P R-C a s 9})$ and suspended at a concentration of $200 \mu \mathrm{M}$ in nuclease free water. The chimeric CRISPR-Cas9 tracrRNAs were ordered from IDT as RNA Ultramers

331 (https://www.idtdna.com/site/order/oligoentry/index/UltraRNA) with the general sequence

334 (where $\mathrm{rN}=\mathrm{RNA}$ bases comprising the tracrRNA sequence and $\mathrm{N}=\mathrm{DNA}$ bases comprising the DNA donor portion ) and suspended at a concentration of $200 \mu \mathrm{M}$ in nuclease free water. The specific Donorguide sequences used in this study can be found in Supplemental Table 1 .To assemble the Donorguide for in

337 vitro studies, $3 u$ l of $200 \mu \mathrm{M}$ crRNA was combined with $3 \mu \mathrm{l}$ of $200 \mu \mathrm{M}$ chimeric tracrRNA and heated to 33895 o C for 5 minutes and immediately removed to incubate on the bench top at RT for 10-20 minutes until crRNA:tracrRNA mix reached RT. $5 \mu$ l of the $100 \mu \mathrm{M}$ crRNA:tracrRNA mix was added to a new nuclease

340 free PCR tube. $4 \mu$ l of Alt-R Cas9 Nuclease 3NLS (62 $\mu \mathrm{M}$ stock) (IDT cat \# 1074181 1074182) was added to 341 bring to a final volume of $9 \mu \mathrm{l}$ and incubated at RT for 20-30 minutes to form the Donorguide RNP 342 complex. 


\section{Transfections}

For Donorguide HEK293T electroporations, 9ul of the preformed Donorguide RNP complex (see above) was diluted with $16 \mu$ in EP buffer (Etta Biotech) to bring to a final volume of $25 \mu$. Diluted Donorguide RNP complex was added to $300 \mu \mathrm{l}$ HEK293T cells in EP buffer at a concentration of 20E6cells $/ \mathrm{ml}$, mixed thoroughly, and $107 \mu \mathrm{l}$ of the HEK293T cell+ Donorguide cocktail was added to each cuvette.

For the TALEN dCas9 Donorguide electroporations, $9 \mu$ l of the preformed dCas 9 Donorguide RNP cocktail and $5 \mu$ l of $100 \mu \mathrm{M}$ ssODN with $4 \mu \mathrm{I}$ PBS was brought to $25 \mu$ l with $16 \mu$ l of EP buffer. Diluted Donorguide RNP complex or ssODN mix was added to $300 \mu$ HEK293T cells in EP buffer at a concentration of $20 \mathrm{E} 6 \mathrm{cells} / \mathrm{ml}$, mixed thoroughly, and $107 \mu \mathrm{l}$ of the HEK293T cell+ Donorguide cocktail was equally distributed to 3 Eppendorf tubes. 2.5 $\mathrm{g}$ of each left and right TALEN arm plasmids were added to each tube and then transferred to an electroporation cuvette.

HEK293T cells were then electroporated with Etta H1 electroporator (Etta Biotech) with the following parameters: $200 \mathrm{~V}, 784 \mathrm{~ms}$ interval, six pulses, and 1,000 $\mu$ s pulse duration. Cells are recovered post

357 electroporation by incubating at $37^{\circ} \mathrm{C}$ for $5-10 \mathrm{~min}$ before being transferred to a six-well tissue cell 358 culture plate at a density of about $1.5 \mathrm{E} 6$ cells $/ \mathrm{mL}$.

\section{DNA isolation and PCR Amplification}

DNA from whole-cell populations was purified using the Qiagen DNeasy Blood \& Tissue Kit (Qiagen 69504). DNA was isolated from zebrafish as described below. PCR amplification was conducted with

362 Platinum SuperFi DNA Polymerase (Thermo Fisher Scientific 12351010) or Q5 ${ }^{\circledR}$ High-Fidelity $2 X$ Master 
Samples used for RFLP analysis were amplified with MyTaq ${ }^{\mathrm{TM}}$ Red Mix (Bioline BIO-25043) and subcloned using MyTaq ${ }^{\mathrm{TM}}$ Red Mix and amplification confirmed by agarose gel electrophoresis. The colony PCR amplified products were digested with Avrll (New England Biolabs R0174S) in CutSmart buffer at $37^{\circ} \mathrm{C}$

371 for 3 hours before being resolved with agarose gel electrophoresis.

\section{GeneWiz AmpliconEZ: DNA library preparation and Illumina sequencing}

PCR products were obtained and purified as mentioned above. Illumina partial adapters

375 (Forward sequencing read: 5'-ACACTCTTTCCCTACACGACGCTCTTCCGATCT-3' reverse sequencing read: 5'-GACTGGAGTTCAGACGTGTGCTCTTCCGATCT-3') were added to the 5' end of sequencing primers. DNA library preparations, sequencing reactions, and initial bioinformatics analysis were conducted at process using the NEBNext Ultra DNA Library Prep Kit, following the manufacturer's recommendations

380 (Illumina). End-repaired adapters were ligated after adenylation of the $3^{\prime}$ ends followed by enrichment 
The raw .fasta files obtained from GeneWiz AmpliconEZ were analyzed using CasAnalyzer ${ }^{46}$ to determine indel efficiency and overall HDR events. The raw output from CasAnalyzer was then further analyzed by NGS Analyzer to quantify precise and imprecise HDR events. This script for NGS Analyzer is hosted at https://github.com/srcastillo/Sequencing under a GNU General Public License v3.0.

\section{TALEN design and assembly}

A pair of TALENs against the EGFP gene were designed and generatedusing the FusX assembly system ${ }^{47}$.

The RVDs utilized were $\mathrm{HD}=\mathrm{C}, \mathrm{NN}=\mathrm{G}, \mathrm{NI}=\mathrm{A}, \mathrm{NG}=\mathrm{T}$. The TALEN pair binds the following EGFP gene

sequence, where the spacer region, which is the site of predicted DSBs, is in lower case:

GGCCCACCCTCGTGAccaccctgacctacGGCGTGCAGTGCTTC. TALEN plasmids were electroporated into HEK-

EGFP cells. After electroporation, substantial gene editing in the bulk population was verified.

\section{Zebrafish husbandry}

400 Wild-type (WT) MRF zebrafish (Danio rerio) were maintained in the Mayo Clinic Zebrafish Core Facility.

401 Fish are handled in accordance with standard practices ${ }^{48}$ and guidelines from the Institutional Animal

402 Care and Use Committee (IACUC) (A34513-13-R16, A8815-15). Adult zebrafish are kept in a 9 L (25 - 30

403 fish) or $3 \mathrm{~L}$ housing tanks ( $10-15$ fish) at $28.5^{\circ} \mathrm{C}$ with a light/dark cycle of $14 / 10$ hours. Adult fish pair

404 breeding was set up a day before microinjections, and dividers were removed the following morning.

405 After microinjections, the fertilized embryos were transferred to Petri dishes (100 mm) in E2 media (1x;

40615 mM NaCl, 0.5 mM KCl, 1 mM MgSO4, $150 \mu \mathrm{M} \mathrm{KH2PO4,} 50 \mu \mathrm{M} \mathrm{Na} 2 \mathrm{HPO} 4,1$ mM CaCl2, 0.7 mM

$407 \mathrm{NaHCO} 3$ ) and maintained in an incubator at $28.5^{\circ} \mathrm{C}$. 
Sexes are not determined in larval fish ( $2-5$ days post-fertilization (dpf)) in this study and thus potential differences based on sex are not assessed. Zebrafish gonads begin developing around $15 \mathrm{dpf}{ }^{49}$. A multifactorial sexual differentiation process, involving multiple genes and environmental factors, takes place around $25 \mathrm{dpf}$ and is considered completed around $60 \mathrm{dpf}\left({ }^{50-52}\right)$.

\section{DNA oligonucleotide preparation}

All single strand oligonucleotides (ssODNs) used in this study were purchased from IDT (Integrated DNA Technology, Inc., Coralville, IA, USA). Stock was prepared at $100 \mu \mathrm{M}$ in 1x TE and working stock was prepared at $20 \mu \mathrm{M}$ to be used for microinjection or left at $100 \mu \mathrm{M}$ for in vitro studies.

\section{crRNA, tracrRNA, Donorguide, and spCas9 preparation for zebrafish microinjection}

All crRNA (crispr RNA), tracrRNA (transactivating crispr RNA), Donorguide (tracrRNA fused with a DNA cargo sequence flanked by homology arms), and SpCas9 (Streptococcus pyogenes Cas9) were customordered from IDT. Stock was prepared at $20 \mu \mathrm{M}$ in Duplex Buffer (IDT; Cat\#11-05-01-03; 30 mM HEPES, $\mathrm{pH}$ 7.5, $200 \mathrm{mM}$ potassium acetate) for crRNA, tracrRNA, and Donorguide. At the $20 \mu \mathrm{M}$ concentration, crRNA is at $233.9 \mathrm{pg} / \mathrm{nL}$ (protospacer length $20 \mathrm{nt}$ ) and tracrRNA is at $432.7 \mathrm{pg} / \mathrm{hL}$. spCas9 stock was prepared at $23 \mu \mathrm{M}(3.73 \mathrm{ng} / \mathrm{nL}$ ) in PBS (phosphate buffered saline; 1x).

\section{Microinjection}

1-cell stage fertilized zebrafish embryos were harvested and aligned on an agarose plate in E2 media. 3 $\mu \mathrm{L}$ of injection solution was loaded into an injection needle (a broken pulled glass pipette). A $2 \mathrm{~nL}$ of injection solution was delivered into the cell interface layer of the embryo. This injection $(2 \mathrm{~nL})$ delivered crRNA:tracrRNA (or Donorguide):spCas9 complex at approximately $4.52 \mathrm{fmol}$ (52.9 pg):4.52 fmol (97.8 
pg):4.52 fmol (733.1 pg) and ssODN (when needed) at $4 \mathrm{fmol}$. Injected embryos were transferred to a new Petri dish and kept in an incubator at $28.5^{\circ} \mathrm{C}$. Dead and nonviable embryos were counted and

432 removed the next day (1 dpf).

\section{Zebrafish experimental replicates}

434 All experiments involving zebrafish microinjection were carried out in three independent experiments.

435 Each experiment utilized a mixed populations of embryos obtained from multiple clutches. Each

436 experiment was ideally conducted on different days. If two experiments were performed on a day, all

437 reagents were prepared from different stock preparations.

\section{Zebrafish genomic DNA extraction}

441 Usually 40 embryos were individually collected in $36 \mu \mathrm{L}$ of $\mathrm{NaOH}(50 \mathrm{mM}$ ) on 1-2 dpf (if morphological

442 phenotyping was not necessary) and on 4-5 dpf (if morphology was assessed). Each embryo in $\mathrm{NaOH}$

443 was heated at $95^{\circ} \mathrm{C}$ for 15 min while vortexing a few times (REF\#6-8). To stabilize $\mathrm{NaOH}, 4 \mu \mathrm{L}$ of tris (1

$444 \mathrm{mM}$ ) was added. 8 embryos were pulled into a sample (a biological replicate) by taking $2 \mu \mathrm{L}$ from each

445 embryo (8 fish per sample), and a 1:3 dilution was made by adding $48 \mu \mathrm{L}$ of distilled water. 
bioRxiv preprint doi: https://doi.org/10.1101/2021.05.28.446234; this version posted May 28, 2021. The copyright holder for this preprint (which

was not certified by peer review) is the author/funder, who has granted bioRxiv a license to display the preprint in perpetuity. It is made available under aCC-BY-NC 4.0 International license.

\section{$448 \quad$ Author contributions}

449 SCE, KJC, HA, and BWS conceived the study. BWS, KJC, SCE, and HBL wrote the manuscript. HA

450 performed proof of concept zebrafish experiments. CD and HBL performed zebrafish experiments. BWS

451 performed cell line experiments and analyzed all data sets. SRC wrote the NGS Analyzer script and

452 assisted with figure and TALEN design. GMG designed the figures. WAG provided preliminary cell line

453 data.

454

455 


\section{Acknowledgements}

457 We would like to thank the Mayo Clinic Zebrafish Facility staff for assisting with animal husbandry and

458 the Mayo Clinic Fluorescent Microscopy core for assistance with flow cytometry. We would also like to

459 thank Dr. Alex Abel for assistance with flow cytometry analysis. We would like to thank Sharoon Akhtar

460 and Xiyin Wang for insightful discussion that informed the experiments. We additionally thank Dr.

461 Maura McGrail and Dr Jeff Essner for their constructive collaboration and advice. This work was

462 supported by NIH R24 OD020166 (SCE, KJC), Mayo Foundation for Medical Education and Research

463 (BWS, SRC,WAG,GMG), Mayo Clinic Regenerative Sciences Training Program (BWS), and the Harry C.

464 and Debra A. Stonecipher Predoctoral Fellowship (SRC).

465

466

467 


\section{References}

$470 \quad 1 . \quad$ Doudna JA, Charpentier E. The new frontier of genome engineering with CRISPR-Cas9. Science. 471 2014;346:1258096. DOI: 10.1126/science.1258096

472 2. Jinek M, Chylinski K, Fonfara I et al. A Programmable Dual-RNA-Guided DNA Endonuclease in

473 Adaptive Bacterial Immunity. Science. 2012;337:816-821. DOI: 10.1126/science.1225829

$474 \quad 3 . \quad$ Ran FA, Hsu PD, Wright J et al. Genome engineering using the CRISPR-Cas9 system. Nature

475 Protocols. 2013;8:2281-2308. DOI: 10.1038/nprot.2013.143

476 4. Mao Z, Bozzella M, Seluanov A et al. Comparison of nonhomologous end joining and

477 homologous recombination in human cells. DNA repair. 2008;7:1765-1771. DOI:

$478 \quad 10.1016 /$ j.dnarep.2008.06.018

479 5. Bae S, Kweon J, Kim HS et al. Microhomology-based choice of Cas9 nuclease target sites. Nature 480 methods. 2014;11:705-706. DOI: 10.1038/nmeth.3015

481 6. Ata H, Ekstrom TL, Martínez-Gálvez G et al. Robust activation of microhomology-mediated end joining for precision gene editing applications. PLoS genetics. 2018;14:e1007652. DOI: 10.1371/journal.pgen.1007652

$4847 . \quad$ Richardson CD, Kazane KR, Feng SJ et al. CRISPR-Cas9 genome editing in human cells occurs via 485 the Fanconi anemia pathway. Nature genetics. 2018;50:1132-1139. DOI: 10.1038/s41588-018-0174-0

486 8. Nakanishi K, Yang YG, Pierce AJ et al. Human Fanconi anemia monoubiquitination pathway promotes homologous DNA repair. Proceedings of the National Academy of Sciences of the United States of America. 2005;102:1110-1115. DOI: 10.1073/pnas.0407796102

489 9. Davis L, Maizels N. Homology-directed repair of DNA nicks via pathways distinct from canonical double-strand break repair. Proceedings of the National Academy of Sciences of the United States of America. 2014;111:E924-932. DOI: 10.1073/pnas.1400236111

10. Hustedt N, Durocher D. The control of DNA repair by the cell cycle. Nat Cell Biol. 2016;19:1-9. DOI: $10.1038 / \mathrm{ncb} 3452$

494 11. Lin S, Staahl BT, Alla RK et al. Enhanced homology-directed human genome engineering by controlled timing of CRISPR/Cas9 delivery. eLife. 2014;3:e04766. DOI: 10.7554/eLife.04766

12. Bothmer A, Phadke T, Barrera LA et al. Characterization of the interplay between DNA repair and CRISPR/Cas9-induced DNA lesions at an endogenous locus. Nature Communications. 2017;8:13905. DOI: 10.1038/ncomms13905

499 13. Chen F, Pruett-Miller SM, Huang $Y$ et al. High-frequency genome editing using ssDNA

500 oligonucleotides with zinc-finger nucleases. Nature methods. 2011;8:753-755. DOI: 10.1038/nmeth.1653

501 14. DeWitt MA, Magis W, Bray NL et al. Selection-free genome editing of the sickle mutation in human adult hematopoietic stem/progenitor cells. Science translational medicine. 2016;8:360ra134. DOI: 10.1126/scitransImed.aaf9336

504 15. Richardson CD, Ray GJ, DeWitt MA et al. Enhancing homology-directed genome editing by 505 catalytically active and inactive CRISPR-Cas9 using asymmetric donor DNA. Nature biotechnology.

506 2016;34:339-344. DOI: 10.1038/nbt.3481

507 16. Liang X, Potter J, Kumar S et al. Enhanced CRISPR/Cas9-mediated precise genome editing by 508 improved design and delivery of gRNA, Cas9 nuclease, and donor DNA. Journal of biotechnology.

509 2017;241:136-146. DOI: 10.1016/j.jbiotec.2016.11.011

510 17. Orlando SJ, Santiago Y, DeKelver RC et al. Zinc-finger nuclease-driven targeted integration into 511 mammalian genomes using donors with limited chromosomal homology. Nucleic acids research.

512 2010;38:e152. DOI: 10.1093/nar/gkq512

513 18. Bedell VM, Wang Y, Campbell JM et al. In vivo genome editing using a high-efficiency TALEN

514 system. Nature. 2012;491:114-118. DOI: 10.1038/nature11537 
19. Li M, Zhao L, Page-McCaw PS et al. Zebrafish Genome Engineering Using the CRISPR-Cas9 System. Trends Genet. 2016;32:815-827. DOI: 10.1016/j.tig.2016.10.005

20. Ceasar SA, Rajan V, Prykhozhij SV et al. Insert, remove or replace: A highly advanced genome editing system using CRISPR/Cas9. Biochim Biophys Acta. 2016;1863:2333-2344. DOI: 10.1016/j.bbamcr.2016.06.009

21. Paix A, Folkmann A, Goldman DH et al. Precision genome editing using synthesis-dependent repair of Cas9-induced DNA breaks. Proceedings of the National Academy of Sciences. 2017;114:E10745E10754. DOI: 10.1073/pnas.1711979114

22. Boel A, De Saffel H, Steyaert W et al. CRISPR/Cas9-mediated homology-directed repair by ssODNs in zebrafish induces complex mutational patterns resulting from genomic integration of repairtemplate fragments. Disease Models \&amp; Mechanisms. 2018;11:dmm035352. DOI: 10.1242/dmm.035352

23. Aird EJ, Lovendahl KN, St Martin A et al. Increasing Cas9-mediated homology-directed repair efficiency through covalent tethering of DNA repair template. Communications biology. 2018;1:54. DOI: 10.1038/s42003-018-0054-2

24. Savic $\mathrm{N}$, Ringnalda FC, Lindsay $\mathrm{H}$ et al. Covalent linkage of the DNA repair template to the CRISPR-Cas9 nuclease enhances homology-directed repair. eLife. 2018;7. DOI: 10.7554/eLife.33761 25. Komor AC, Kim YB, Packer MS et al. Programmable editing of a target base in genomic DNA without double-stranded DNA cleavage. Nature. 2016;533:420-424. DOI: 10.1038/nature17946 26. Gaudelli NM, Komor AC, Rees HA et al. Programmable base editing of $A^{*} T$ to $G^{*} C$ in genomic DNA without DNA cleavage. Nature. 2017;551:464-471. DOI: 10.1038/nature24644

27. Anzalone AV, Randolph PB, Davis JR et al. Search-and-replace genome editing without doublestrand breaks or donor DNA. Nature. 2019;576:149-157. DOI: 10.1038/s41586-019-1711-4

28. Papapetrou EP, Schambach A. Gene Insertion Into Genomic Safe Harbors for Human Gene Therapy. Molecular therapy : the journal of the American Society of Gene Therapy. 2016;24:678-684. DOI: $10.1038 / \mathrm{mt} .2016 .38$

29. Glaser A, McColl B, Vadolas J. GFP to BFP Conversion: A Versatile Assay for the Quantification of CRISPR/Cas9-mediated Genome Editing. Molecular Therapy - Nucleic Acids. 2016;5:e334. DOI: https://doi.org/10.1038/mtna.2016.48

30. Krug II RG, Poshusta TL, Skuster KJ et al. A transgenic zebrafish model for monitoring glucocorticoid receptor activity. Genes, Brain and Behavior. 2014;13:478-487. DOI: https://doi.org/10.1111/gbb.12135

31. Jao L-E, Wente SR, Chen W. Efficient multiplex biallelic zebrafish genome editing using a CRISPR nuclease system. Proceedings of the National Academy of Sciences. 2013;110:13904-13909. DOI: 10.1073/pnas.1308335110

32. Paul B, Montoya G. CRISPR-Cas12a: Functional overview and applications. Biomedical Journal. 2020;43:8-17. DOI: https://doi.org/10.1016/j.bj.2019.10.005

33. Walton RT, Christie KA, Whittaker MN et al. Unconstrained genome targeting with near-PAMless engineered CRISPR-Cas9 variants. Science. 2020;368:290-296. DOI: 10.1126/science.aba8853

34. Okamoto S, Amaishi Y, Maki I et al. Highly efficient genome editing for single-base substitutions using optimized ssODNs with Cas9-RNPs. Scientific Reports. 2019;9:4811. DOI: 10.1038/s41598-01941121-4

35. Nemudryi AA, Valetdinova KR, Medvedev SP et al. TALEN and CRISPR/Cas Genome Editing Systems: Tools of Discovery. Acta Naturae. 2014;6:19-40.

36. Lamb BM, Mercer AC, Barbas CF, 3rd. Directed evolution of the TALE N-terminal domain for recognition of all 5' bases. Nucleic acids research. 2013;41:9779-9785. DOI: 10.1093/nar/gkt754 37. Bedell VM, Wang Y, Campbell JM et al. In vivo genome editing using a high-efficiency TALEN system. Nature. 2012;491:114-118. DOI: 10.1038/nature11537 
38. Ma AC, Lee HB, Clark KJ et al. High efficiency In Vivo genome engineering with a simplified 15RVD GoldyTALEN design. PLoS One. 2013;8:e65259. DOI: 10.1371/journal.pone.0065259

39. Feng $Y$, Zhang $S$, Huang $X$. A robust TALENs system for highly efficient mammalian genome editing. Scientific Reports. 2014;4:3632. DOI: 10.1038/srep03632

40. Renaud J-B, Boix C, Charpentier M et al. Improved Genome Editing Efficiency and Flexibility Using Modified Oligonucleotides with TALEN and CRISPR-Cas9 Nucleases. Cell reports. 2016;14:22632272. DOI: https://doi.org/10.1016/j.celrep.2016.02.018

41. Ma AC, McNulty MS, Poshusta TL et al. FusX: A Rapid One-Step Transcription Activator-Like Effector Assembly System for Genome Science. Human Gene Therapy. 2016;27:451-463. DOI: 10.1089/hum.2015.172

42. Gratz SJ, Cummings AM, Nguyen JN et al. Genome Engineering of Drosophila with the CRISPR RNA-Guided Cas9 Nuclease. Genetics. 2013;194:1029-1035. DOI: 10.1534/genetics.113.152710 43. Hoshijima K, Jurynec Michael J, Grunwald David J. Precise Editing of the Zebrafish Genome Made Simple and Efficient. Developmental Cell. 2016;36:654-667. DOI: https://doi.org/10.1016/j.devcel.2016.02.015

44. Boel A, De Saffel H, Steyaert W et al. CRISPR/Cas9-mediated homology-directed repair by ssODNs in zebrafish induces complex mutational patterns resulting from genomic integration of repairtemplate fragments. Disease Models \& Mechanisms. 2018;11. DOI: 10.1242/dmm.035352

45. Kato GJ, Piel FB, Reid CD et al. Sickle cell disease. Nature Reviews Disease Primers. 2018;4:18010. DOI: $10.1038 / \mathrm{nrdp} .2018 .10$

46. Park J, Lim K, Kim J-S et al. Cas-analyzer: an online tool for assessing genome editing results using NGS data. Bioinformatics. 2016;33:286-288. DOI: 10.1093/bioinformatics/btw561

47. Ma AC, McNulty MS, Poshusta TL et al. FusX: A Rapid One-Step Transcription Activator-Like Effector Assembly System for Genome Science. Hum Gene Ther. 2016;27:451-463. DOI: 10.1089/hum.2015.172

48. Cunliffe VT. Zebrafish: A Practical Approach. Edited by C. NÜSSLEIN-VOLHARD and R. DAHM. Oxford University Press. 2002. 322 pages. ISBN 019963808 X. Price $£ 40.00$ (paperback). ISBN 019 963809 8. Price $\mathrm{f} 80.00$ (hardback). Genetical Research. 2003;82:79-79. DOI: $10.1017 /$ S0016672303216384

49. Hiroya T. Juvenile Hermaphroditism in the Zebrafish, Brachydanio rerio. Bulletin of the Faculty of Fisheries Hokkaido University. 1977;28:57-65.

50. Lau ES-W, Zhang Z, Qin M et al. Knockout of Zebrafish Ovarian Aromatase Gene (cyp19a1a) by TALEN and CRISPR/Cas9 Leads to All-male Offspring Due to Failed Ovarian Differentiation. Scientific Reports. 2016;6:37357. DOI: 10.1038/srep37357

51. Orban L, Sreenivasan R, Olsson PE. Long and winding roads: testis differentiation in zebrafish. Mol Cell Endocrinol. 2009;312:35-41. DOI: 10.1016/j.mce.2009.04.014

52. Uchida D, Yamashita M, Kitano T et al. Oocyte apoptosis during the transition from ovary-like tissue to testes during sex differentiation of juvenile zebrafish. J Exp Biol. 2002;205:711-718. 
Figure 1-Donorguide composition and experimental workflow (Left) Donorguide consists of a canonical CRISPR RNA (crRNA), trans activating crRNA (tracrRNA) that is fused to a DNA repair template (Top) In vitro experiments were carried out by electroporating Donorguide RNP complexes into cells followed by next generation sequencing (Bottom) In vivo experiments were carried out by microinjecting single cell zebrafish embryos with Donorguide RNP complexes cells followed by next generation sequencing

Figure 2- In vitro validation of CRISPR Donorguide at AAVS1 (A) AAVS1 CRISPR Cas9 target site and corresponding CRISPR Donorguide Design. A 2 nt insert was introduced flanked by 12 nt of homolgy to the genomic target site. (B) Quantification of CRISPR Donorguide Knock-in in HEK293T cells. Precise HDR was quantified by CasAnalyzer and then filtered by NGS Analyzer to call precise insertion events corresponding CRISPR Donorguide Design. A 2 nt insert was introduced flanked by 12 nt of homolgy to the genomic target site. (B) Quantification of CRISPR Donorguide Knock-in in zebrafish. Precise HDR was quantified by CasAnalyzer and filtered through NGS Analyzer to call overall and precise HDR events by FACS sorting. HEK EGFP cells were electroporated with CRISPR Donorguide or an ssODN encoding the expression by flow cytometry 72 hours post transfection (B) Donorguide and ssODN design containing

624 the 2 amino acid substitution flanked by 10nt of homology (C) Representative Flow cytometry data of 625 HEK EGFP cells transfected with either WT SpCas9 and an ssODN encoding the EGFP-BFP substitution or 
Figure 5-CRISPR Donorguide Converts EGFP-BFP more efficiently than an ssODN in vivo (A) Schematic

629 diagram of EGFP-BFP conversion in zebrafish. Transgenic zebrafish with EGFP integrated into the

630 genome by tol2 transposition were injected the single cell stage with either CRISPR Donorguide or WT

631 SpCas9 and an ssODN encoding the EGFP-BFP substitution (B) Quantification of EGFP-BFP substitution in

632 vivo. Injected animals were pooled and submitted for next generation sequencing and analyzed

633 by CasAnalyzer and further analyzed by NGSAnalyzer to call precise or imprecise HDR events

Figure 6-CRISPR Donorguide introduces a $2 \mathrm{bp}$ insertion more efficiently than an ssODN in vitro (A) A were introduced into HEK293T cells by Tol2 transposon and sorted for the highest expressing GFP+ cells. 
651 dCas9 Donorguide encoding the EGFP-BFP mutation. Transfected cells were measured for BFP

652 expression 72 hours post transfection.

653 Figure 9- CRISPR Donorguide introduces an Avrll Site at a higher rate than an SsODN in vivo (A)

654 Schematic representation of the tyr locus and Cas9 sgRNA target site. WT tyr zebrafish are pigmented

655 throughout the body and lens (B) Schematic representation of the tyr locus with the Avrll site inserted

656 and generating a premature stop codon. The subsequent nonsense mutation results in albino zebrafish

657 lacking body and lens pigmentation (C-F) Representative images of zebrafish targeted at the tyr locus

658 with either an ssODN or Donorguide (G) Representative image of the Avrll RFLP assay showing the WT

659 amplicon is approximately 580 but when the Avrll site is introduced and facilitates digestion, 2 bands of

660335 and 250bp are generated. (H) Quantification on RFLP activity using either an sSODN or CRISPR

661 Donorguide to introduce the Avrll site in tyr 
Supplemental Figure 1- In vitro validation of SpCas9 sgRNA at AAVS1 (A) CRISPR/Cas9 AAVS1 genomic

664 target site (B) Quantifcation of Cas9 activity at AAVS1 in various cell types by Sanger Sequencing and ICE

665 Analysis

Supplemental Figure 2-Generation of HEK EGFP Reporter Cell line (A) Untransfected HEK 293T cells (B)

667 HEK 293T cells transfected with pkTol2cEGFP plasmid + pkC Tol2 plasmid and passaged 10 times.

668 Gating indicates EGFP cells sorted to generate HEK EGFP cell line

Supplemental Figure 3-Donorguide converts EGFP-BFP in vitro Next Generation Sequencing confirmation of EGFP-BFP transition with CRISPR donorguide or an SSODN in HEK GFP reporter cells.

\section{Supplemental Figure 4-Donorguide introduces an Avrll restriction site at a higher rate than a}

673 with Avrll. 8 clones were excluded for either failing to amplify or amplifying a band the size of an empty

674 strataclone vector. 10 clones successfully digested with Avrll (Bottom two rows) Donorguide injected

675 subclones amplified with colony PCR and digested with Avrll. 7 clones were excluded for failing to 676 amplify 34 clones successfully digested with Avrll. 
Figure 1

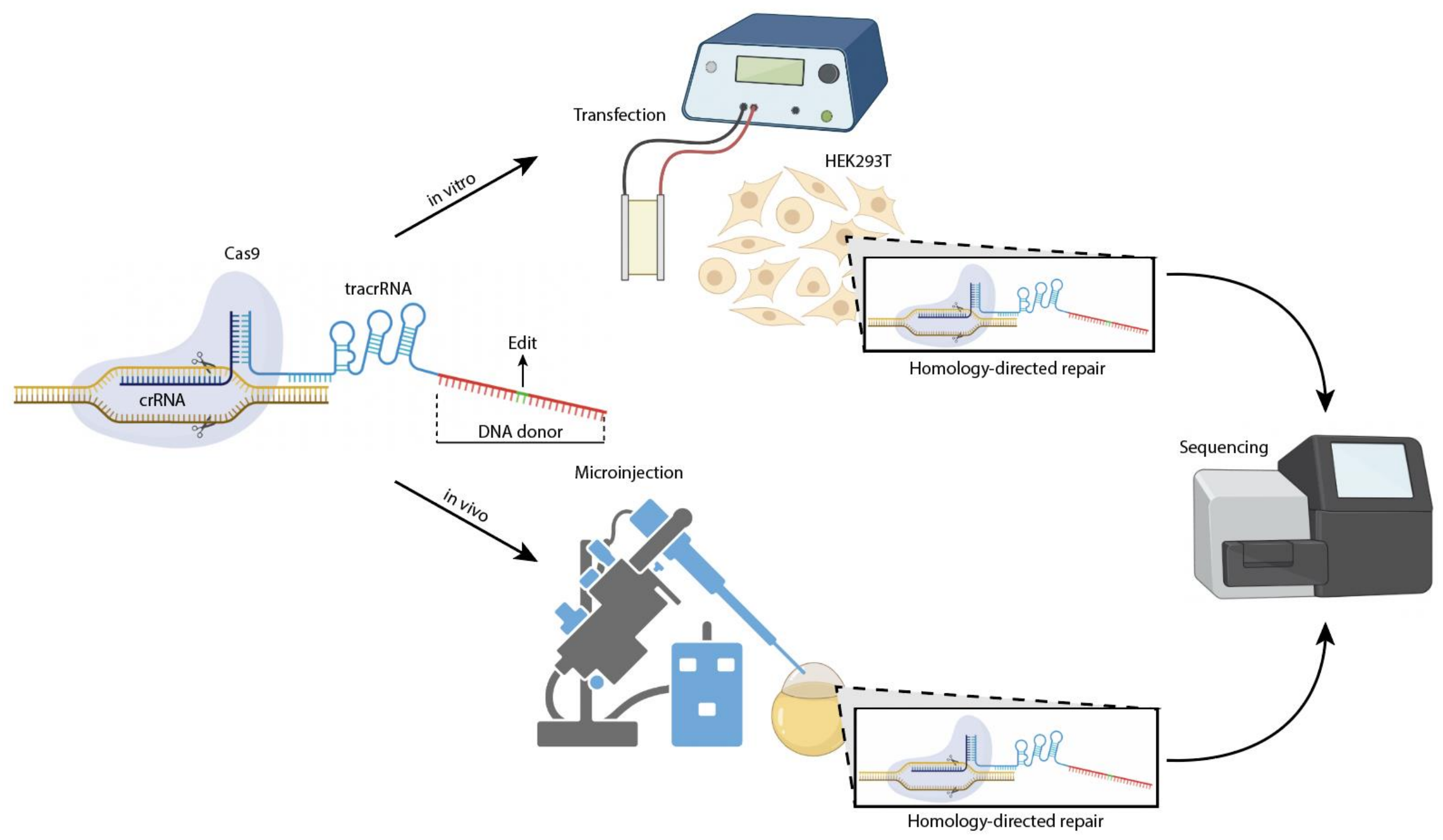




\section{Figure 2}

A.

cagccgcgtcagaagtctcggaggctgccgg

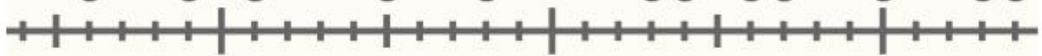
gtcggcgcagtcttcagagcetccgacggcc

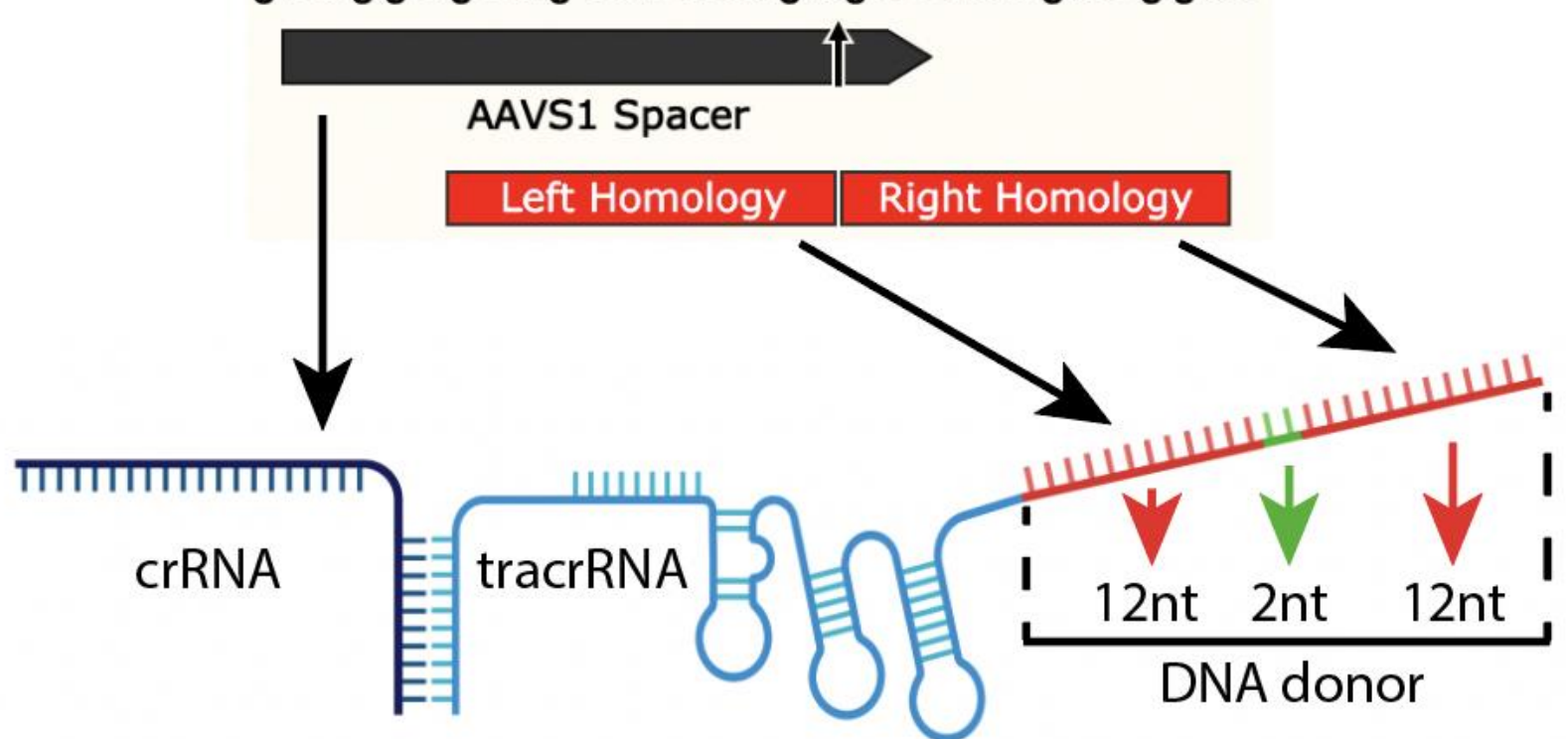

B. CRISPR-Donorguide $2 \mathrm{bp}$ Insertion

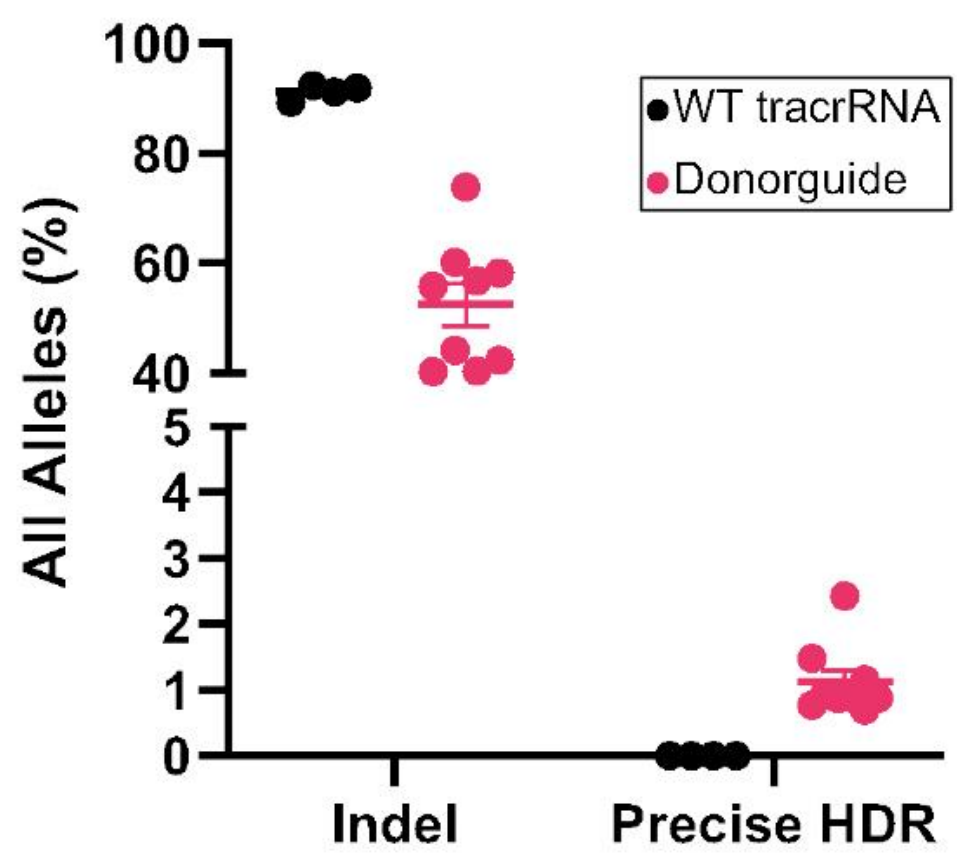


Figure 3

A.

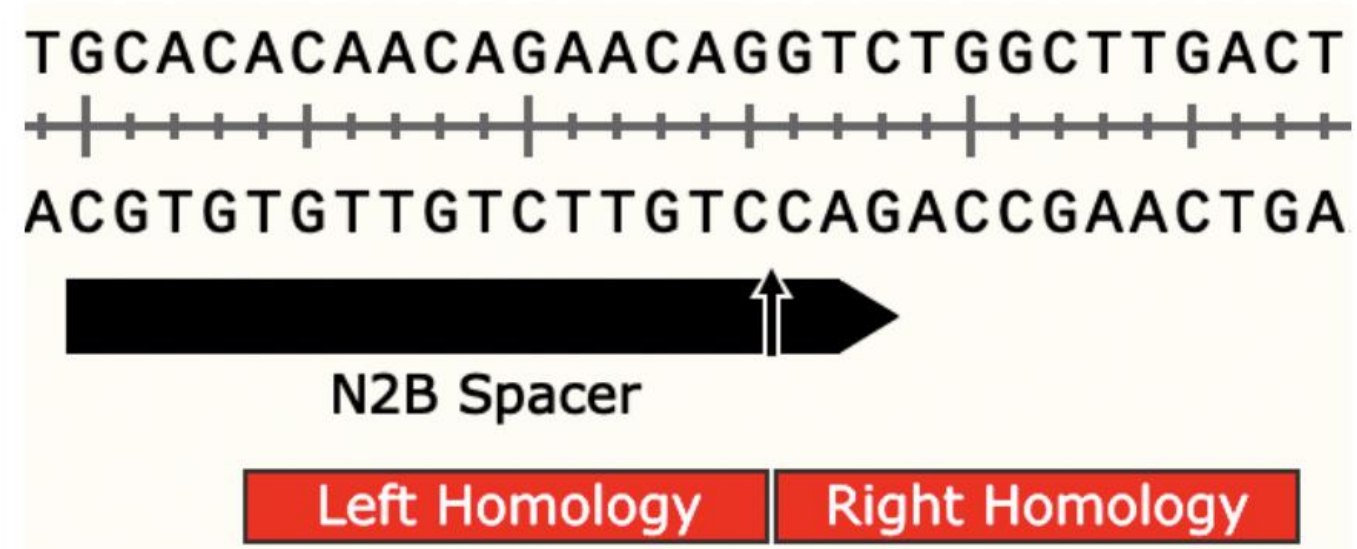

B.

\section{Zebrafish N2B HDR}

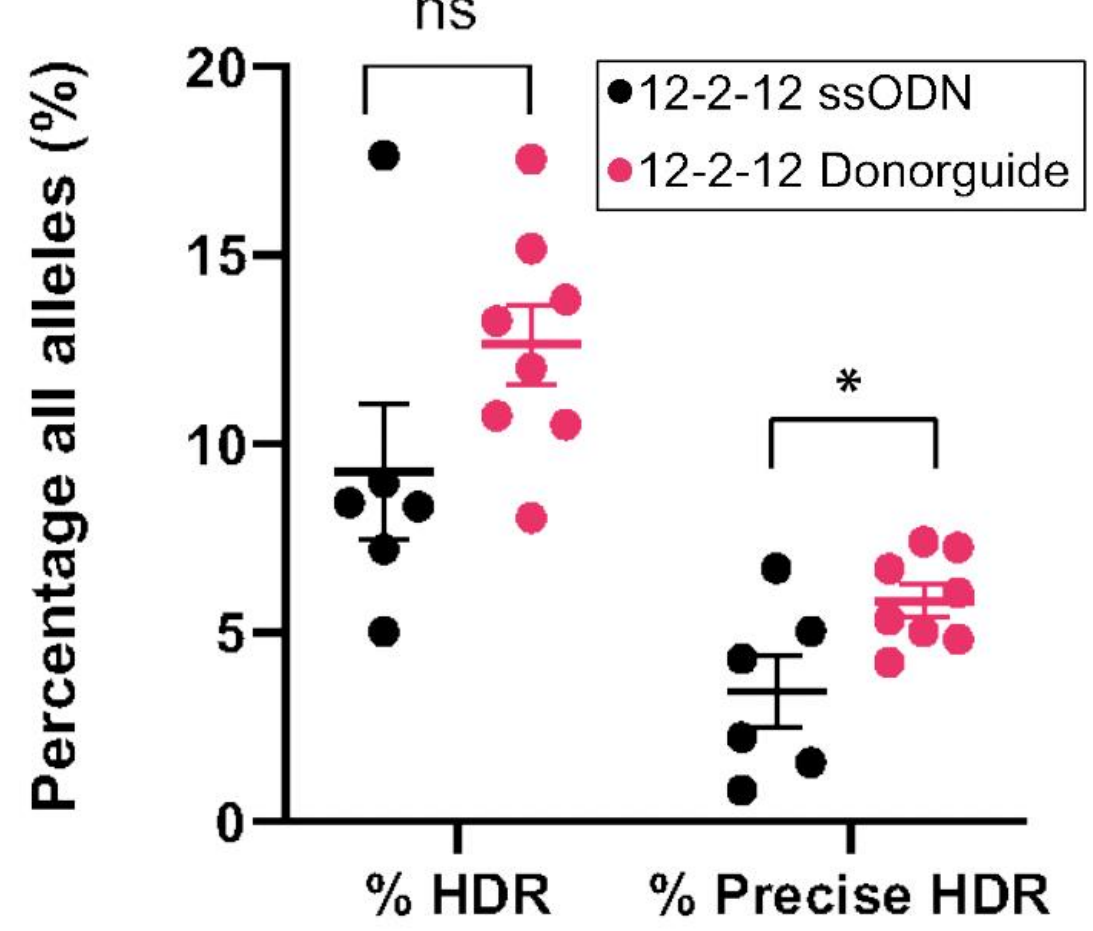


Figure 4

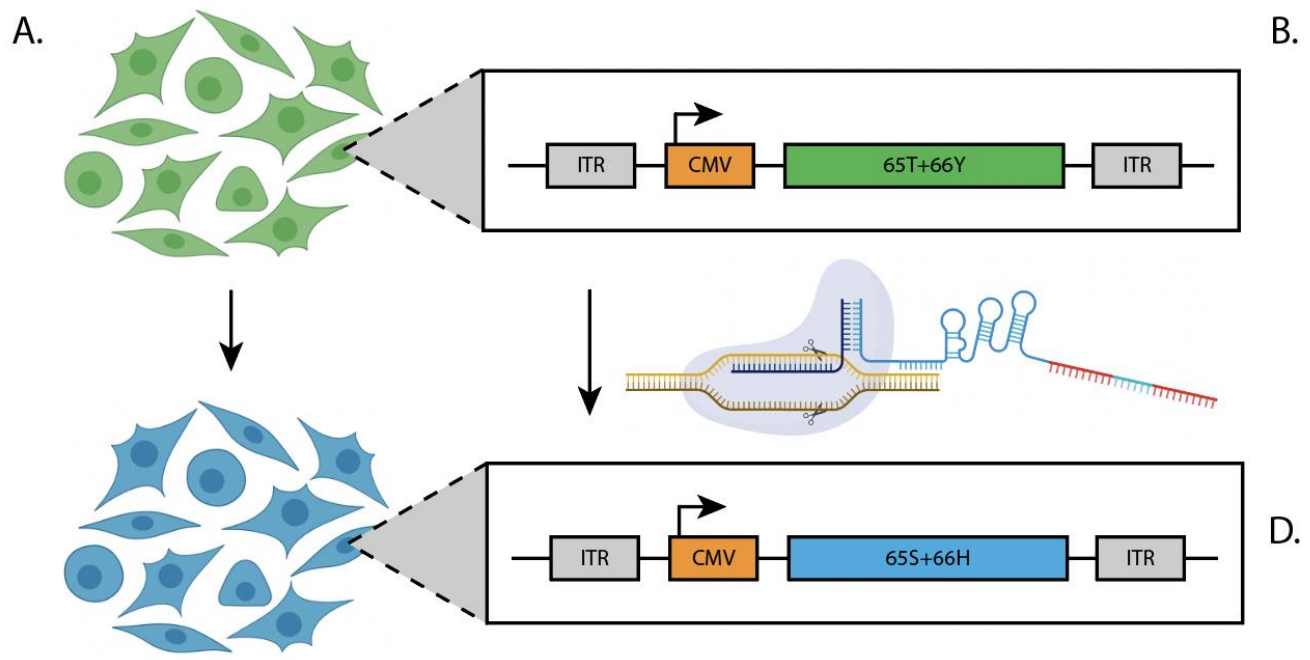

B.
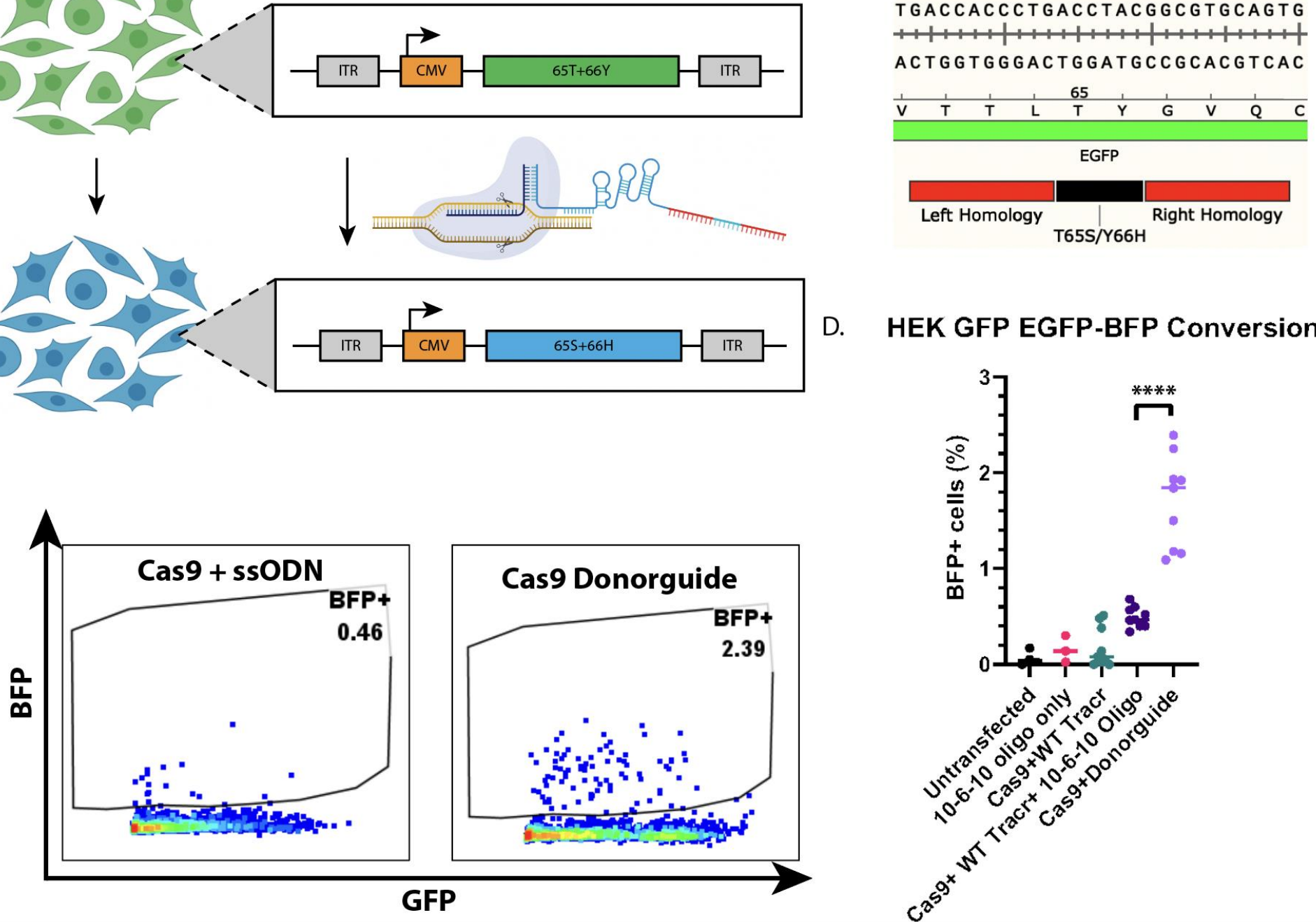

HEK GFP EGFP-BFP Conversion

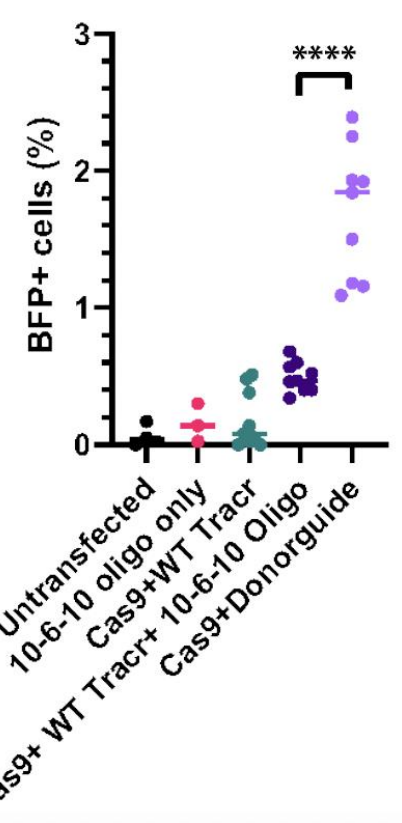


Figure 5

A.
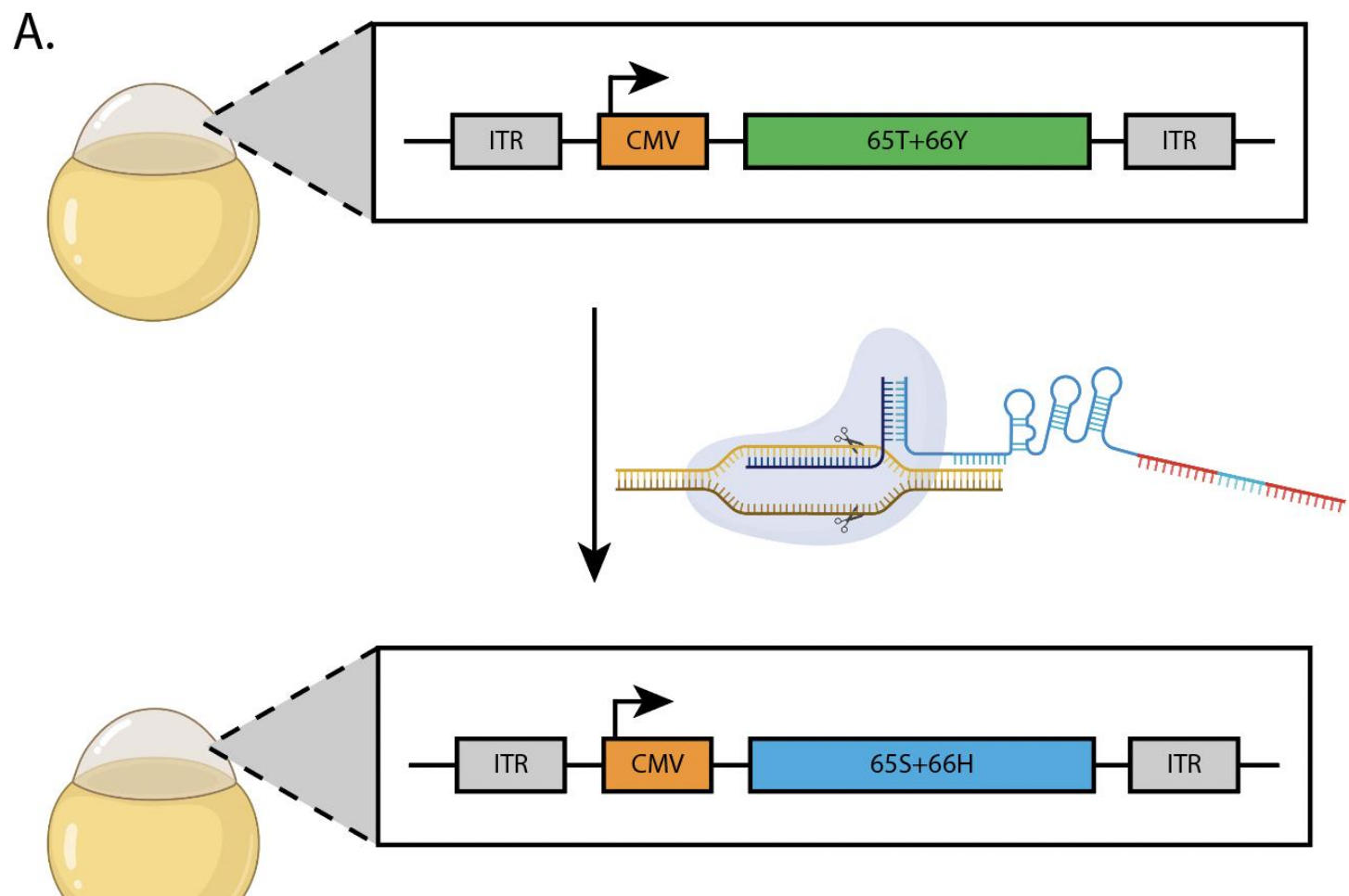

B.

Zebrafish EGFP-BFP Conversion

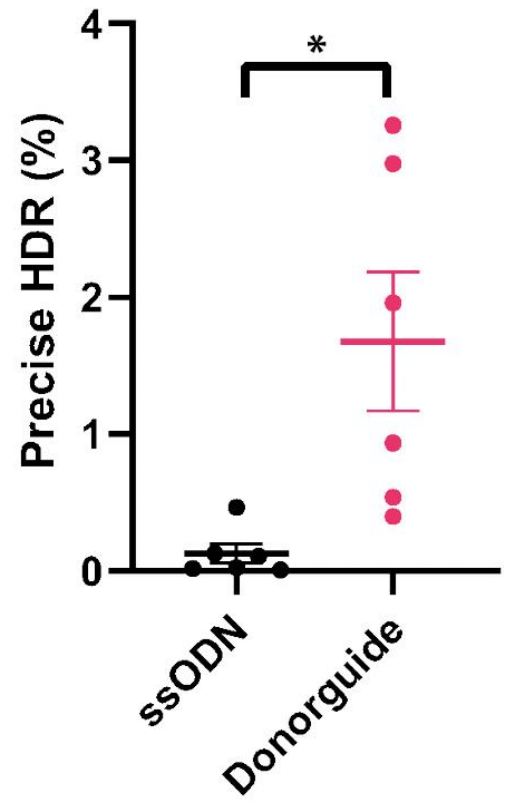




\section{Figure 6}

A.

GTCAGGACAGCCGCGTCAGAAGTCTCGGAGGCTGCCGGTATCTCATTTC

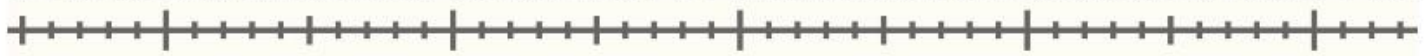
CAGTCCTGTCGGCGCAGTCTTCAGAGCCTCCGACGGCCATAGAGTAAAG Left Homology Right Homology

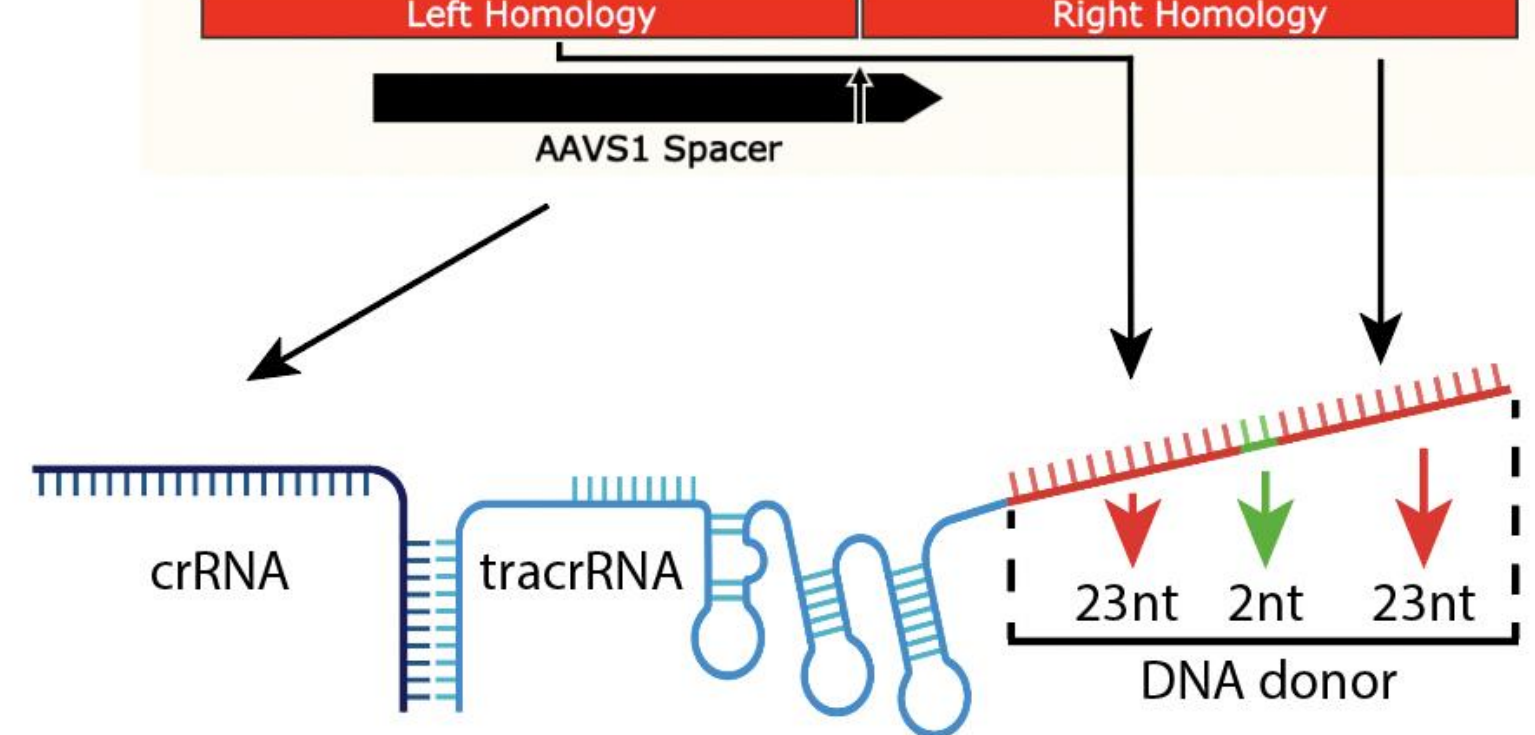

B. HEK AAVS1 Donorguide vs Free ssODN HDR

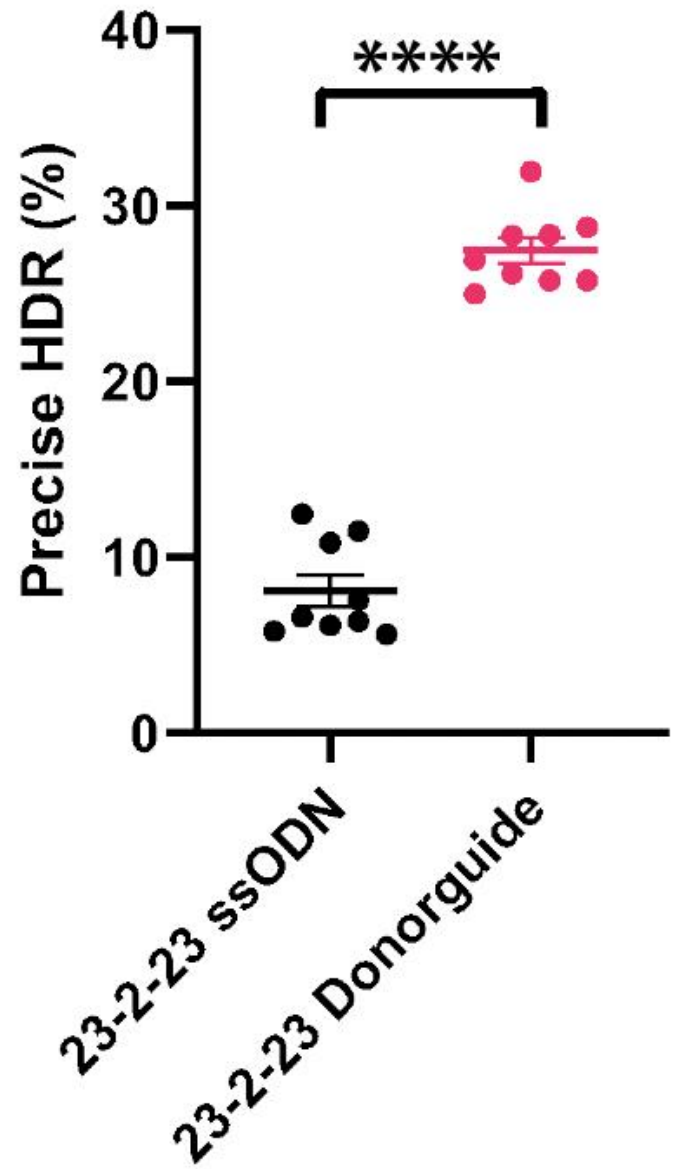


Figure 7

A.

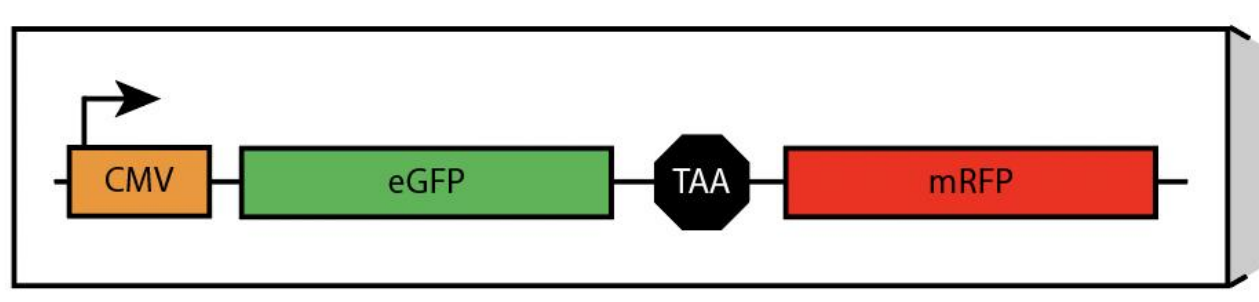

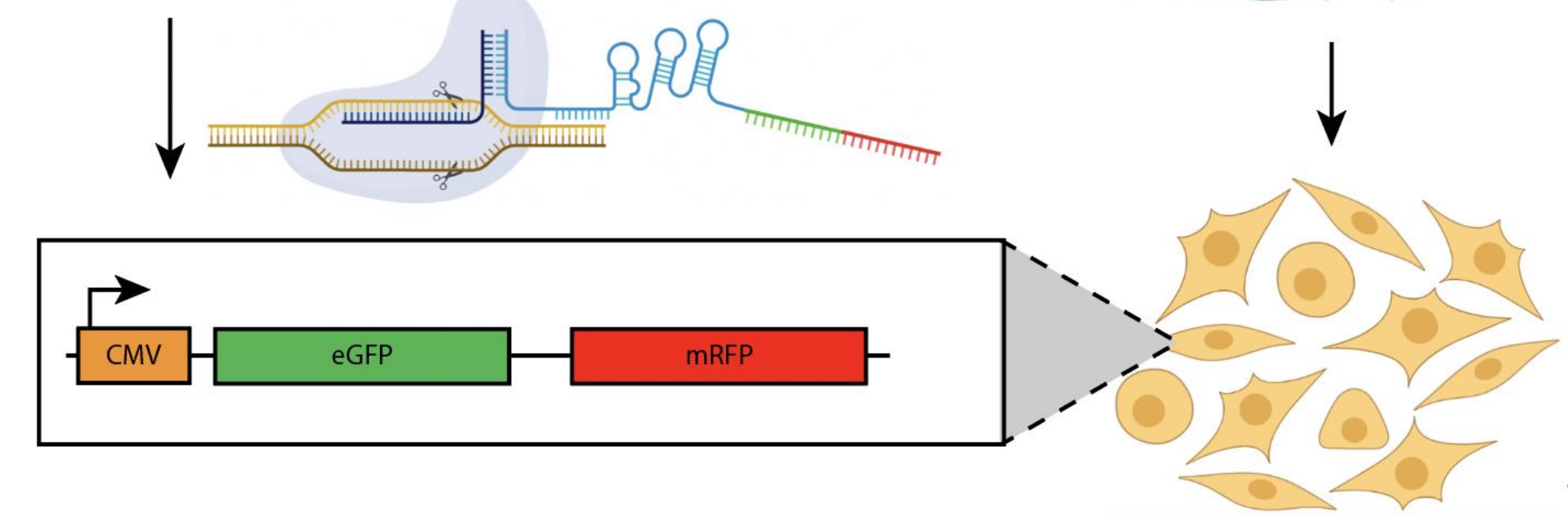

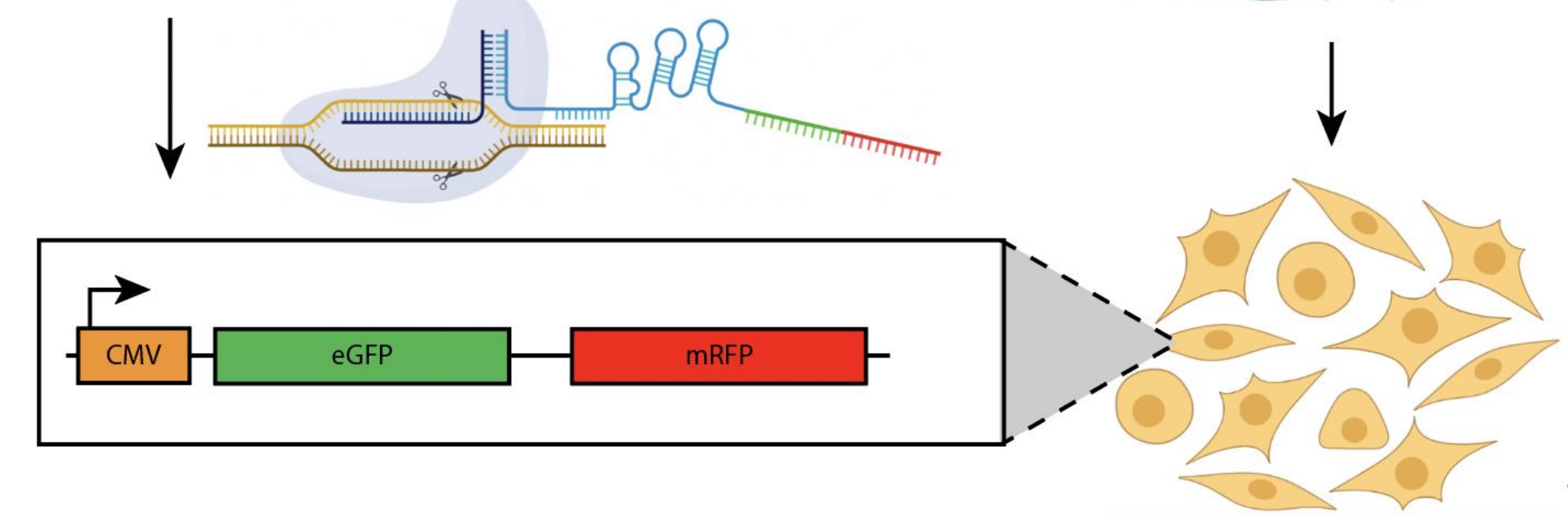

\section{4-0-24 Stop Codon Deletion}

• ssODN

- Donorguide

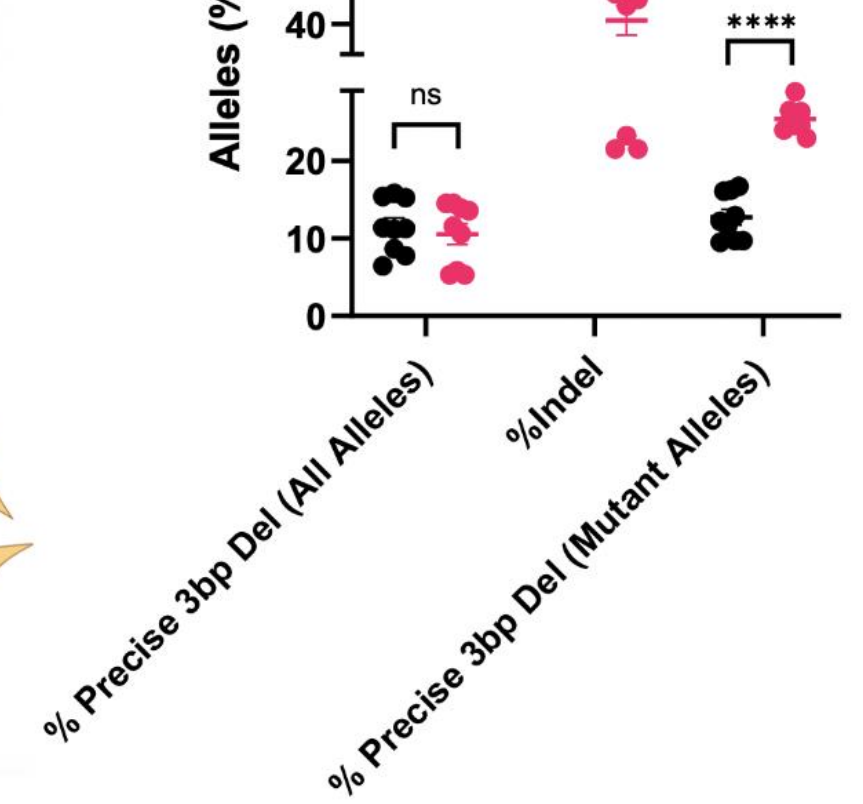




\section{Figure 8}

A.

dCas9

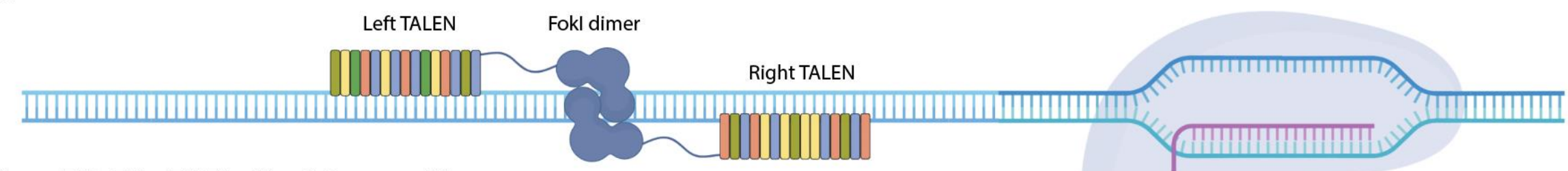

C. TALEN +EGFP dCas9 Donorguide
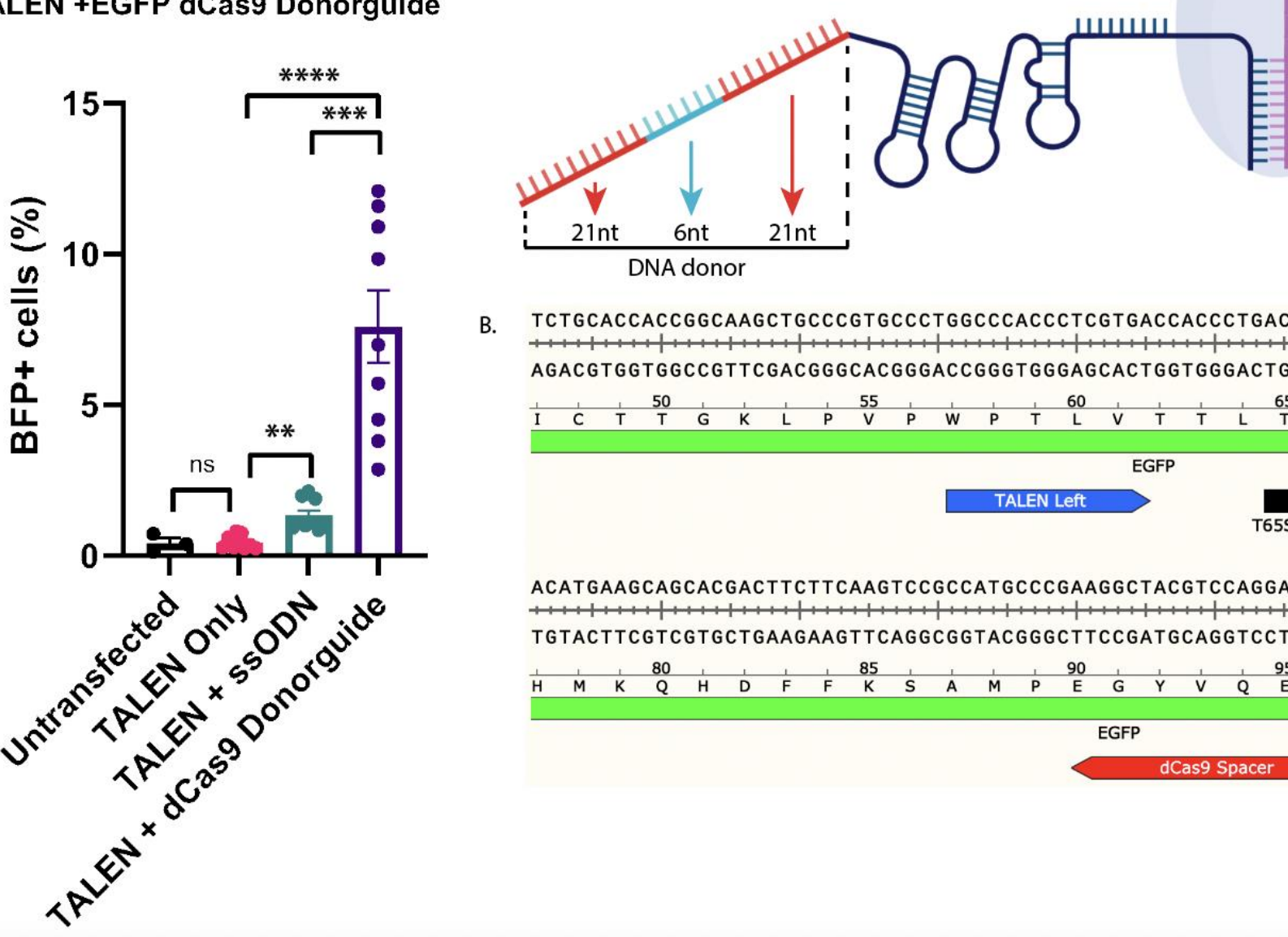

B. TCTGCACCACCGGCAAGCTGCCCGTGCCCTGGCCCACCCTCGTGACCACCCTGACCTACGGCGTGCAGTGCTTCAGCCGCTACCCCGACC AGACGTGGTGGCCGTTCGACGGGCACGGGACCGGGTGGGAGCACTGGTGGGACTGGATGCCGCACGTCACGAAGTCGGCGATGGGGCTGG

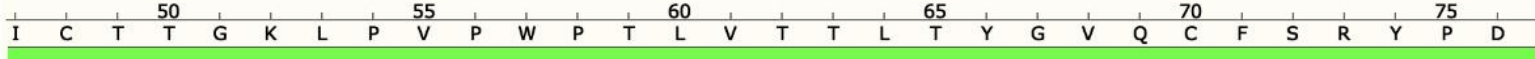

TALEN Right

ACATGAAGCAGCACGACTTCTTCAAGTCCGCCATGCCCGAAGGCTACGTCCAGGAGCGCACCATCTTCTTCAAGGACGACGgCAA TGTACTTCGTCGTGCTGAaGAAGTTCAGGCGGTACGgGCTTCCGATGCAGGTCCTCGCGTGGTAGAAGAAGTTCCTGCTGCCGTT

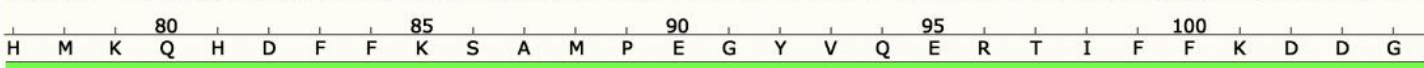




\section{Figure 9}
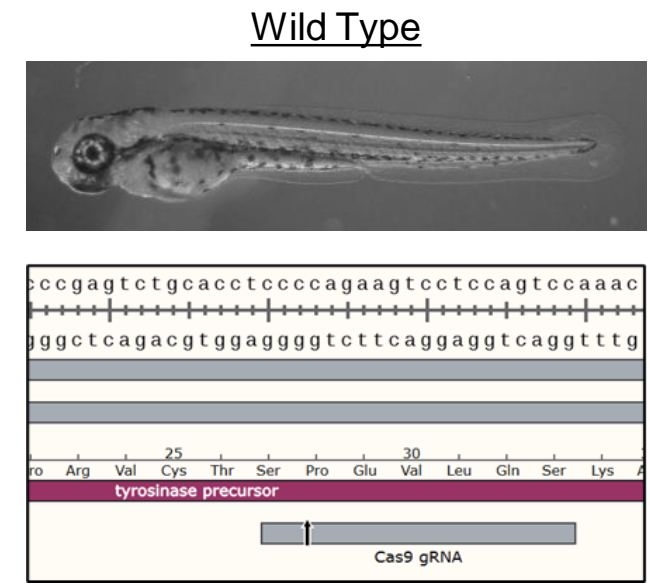

C

Uninjected

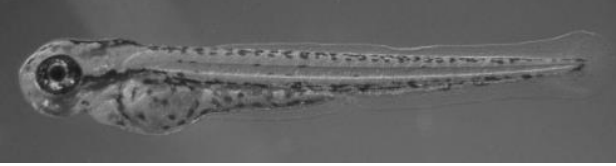

E Donorguide (Complementary Strand)

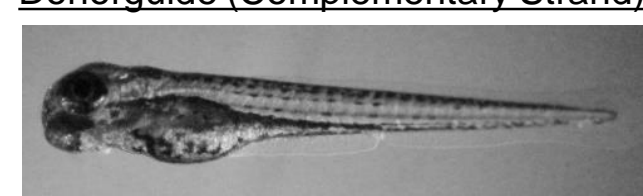

B

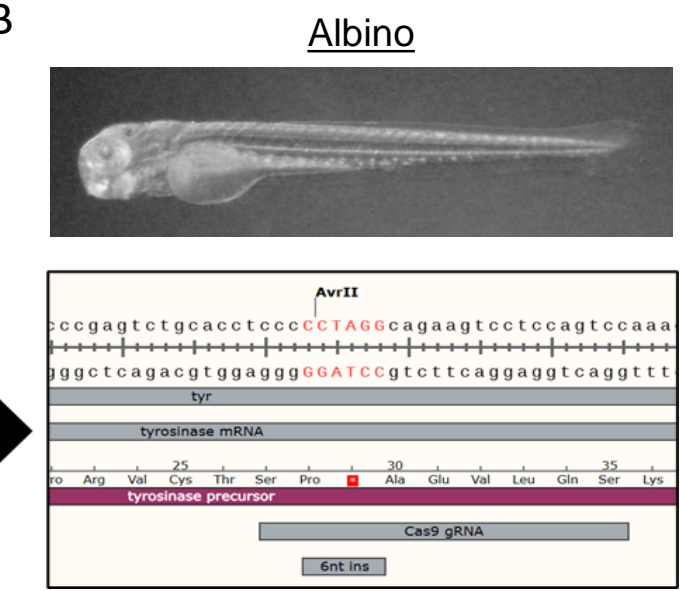

D

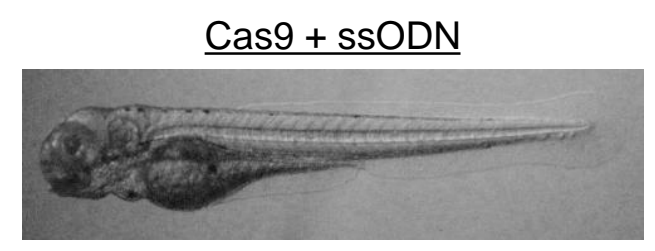

F

$$
\text { Donorguide (Same Strand) }
$$

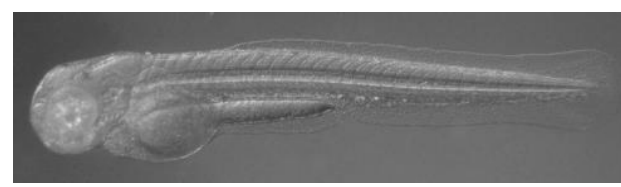

G

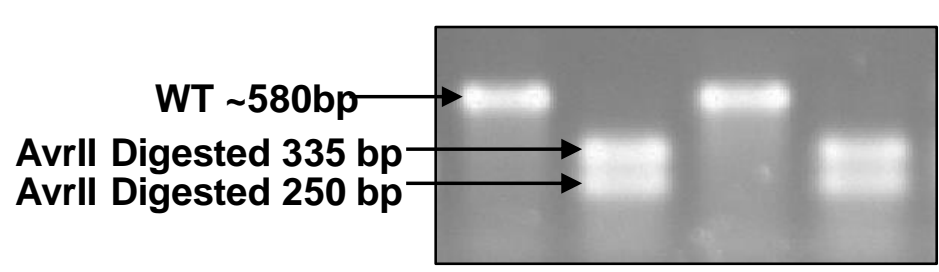

$\mathrm{H}$

Avrll 12-6-12 Tyrosinase ssODN vs DG

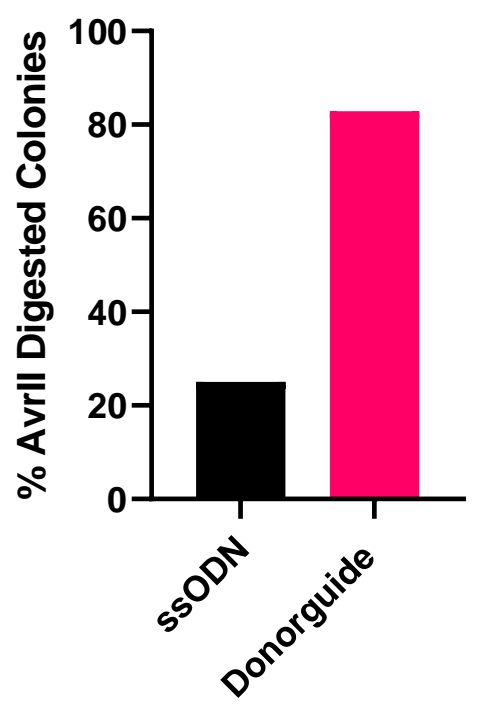

- sSODN

Donorguide 


\section{Supplemental Figure 1}

A

tggccagcggccccgtcaggacAGCCGCGTCAGAAGTCTCGGagg accggtcgccggggcagtcctgTCGGCGCAGTCTTCAGAGCCtcC xccggtcgCcggggcagtcCtgTCGGCGCAGTCTTCAGAGCCLCC PPP1R12C
B

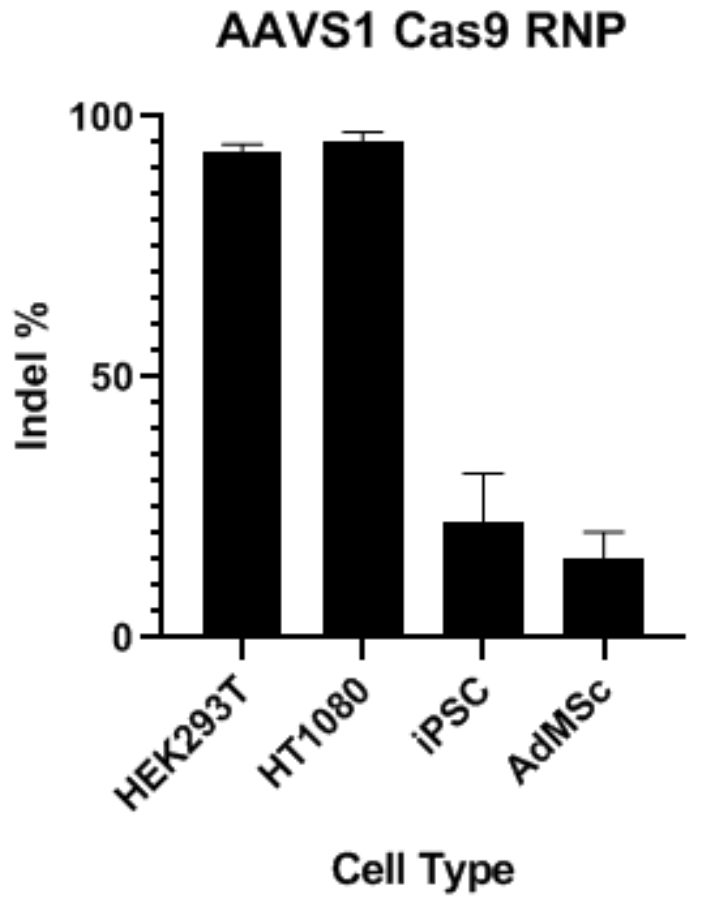




\section{Supplemental Figure 2}



B

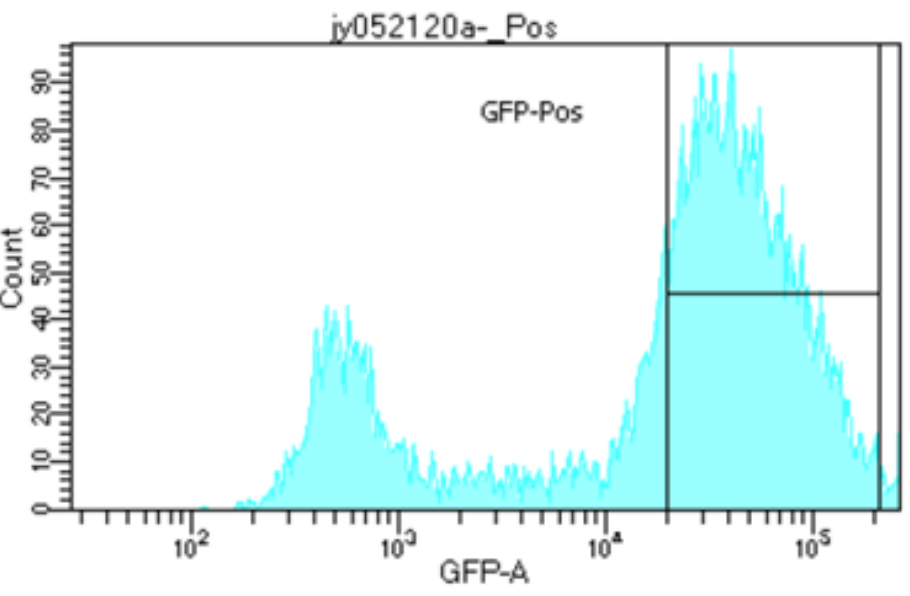




\section{Supplemental Figure 3}

EGFP-BFP Conversion

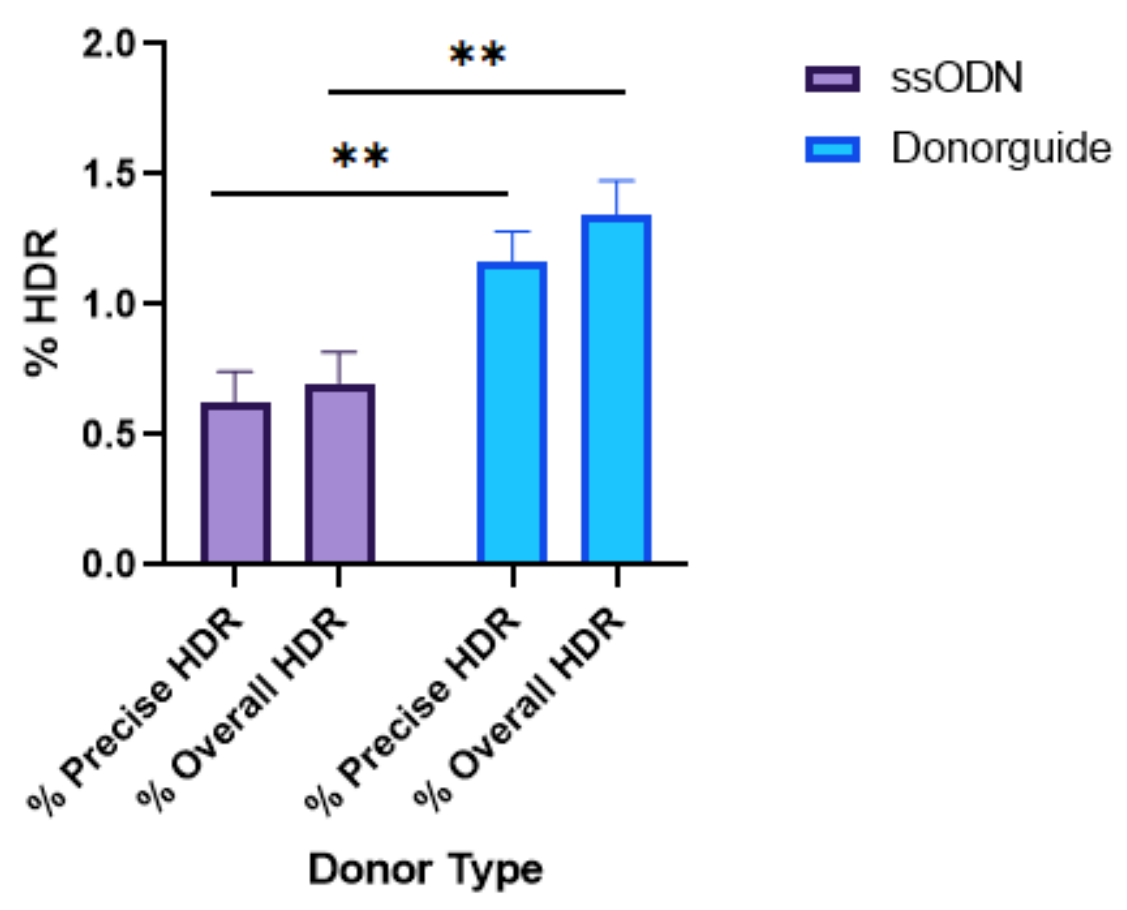




\section{Supplemental Figure 4}

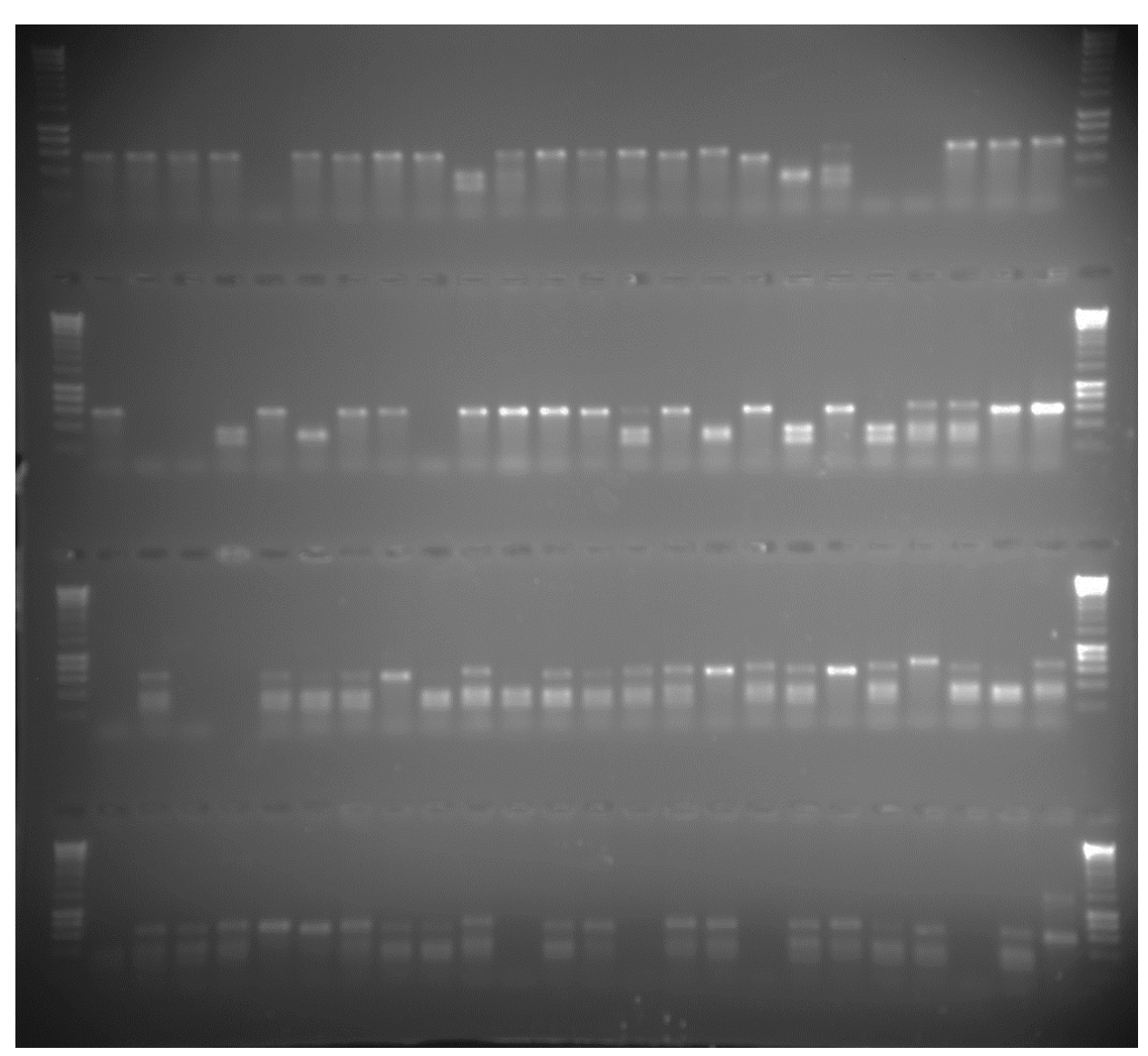

